\title{
Research Paper \\ Effect of Kyphosis Exercises on Physical Function, Postural Control and Quality of Life in Elderly Men With Hyperkyphosis
}

\author{
${ }^{*}$ Aynollah Naderi ${ }^{1} \odot$, Mohammad Hossain Rezvani ${ }^{1}$, Fatemeh Shaabani ${ }^{2}$, , Shahabeddin Bagheri ${ }^{3}$ \\ 1. Department of Biological Sports, Faculty of Physical Education and Sport Sciences, Shahrood University of Technology, Shahrood, Iran. \\ 2. Department of Motor Behaviour and Sports Psychology, Faculty of Physical Education and Sport Sciences, Allameh Tabataba'i University, Tehran, Iran. \\ 3. Department of Sport Injury and Corrective Exercise, Faculty of Physical Education, University of Nahavand, Hamadan, Iran.
}

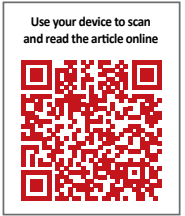

Citation Naderi A, Rezvani MH, Shaabani F, Bagheri Sh. [Effect of Kyphosis Exercises on Physical Function, Postural Control and Quality of Life in Elderly Men With Hyperkyphosis (Persian)]. Iranian Journal of Ageing. 2019; 13(4):464-479. https://doi.org/10.32598/SIJA.13.4.464

\section{https://doi.org/10.32598/SIJA.13.4.464}

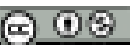

Received: 12 May 2018 Accepted: 27 Oct 2018 Available Online: 01 Jan 2019

Keywords:

Kyphosis, Quality of Life, Postural control, Exercise therapy, Physical readiness

\section{A B STRACT}

Objectives The present study investigated the effect of kyphosis correction exercise program on physical function, balance, and the quality of life in older men with hyperkyphosis.

Methods \& Materials Twenty-four men with kyphosis of $\geq 50^{\circ}$ were randomly assigned into an intervention group $(n=12$, with Mean $\pm S D$ age $=68.75 \pm 2.67$ years, Mean $\pm S D$ height $=173.66 \pm 3.75 \mathrm{~cm}$, Mean $\pm S D$ weight $=73.5 \pm 4.75 \mathrm{~kg})$, and a control group $(n=12$, with Mean $\pm S D$ age $=69.5 \pm 2.64$ years, Mean $\pm S D$ height $=174.4 \pm 7.78 \mathrm{~cm}$, Mean $\pm S D$ weight $=75.41 \pm 5.21 \mathrm{~kg}$ ). The intervention group attended the corrective exercises program, consisting of 6 exercises that were conducted in 3 sessions per week for 12 weeks. Thoracic kyphosis was assessed using flexible ruler and measuring distance between $T_{2}-T_{12}$ vertebras. Quality of life, static balance on both the eyes open and eyes closed and physical performance were assessed using 36-item Short Form Health Survey, sharpened Romberg test, and 6-minute walk test, respectively. Analysis of Covariance (ANCOVA) was used to analyze the obtained data, at the significance level of $\mathrm{P}<0.05$

Results The obtained results suggest that kyphosis corrective exercises program have significant positive effects on physical function, balance, and movement confidence in the elderly people $(P<0.05)$. In addition, after implementing 12 weeks of kyphosis corrective exercises, physical pain, physical function/ limitations, public and mental health were significantly improved $(P<0.05)$. While other dimensions of the quality of life, showed no significant changes $(P>0.05)$.

Conclusion Based on the study results, kyphosis corrective protocol can improve physical function, balance and the quality of life. It could also reduce the economic burden of the disease, and complications related to aging in older men with kyphosis $\leq 50^{\circ}$. Therefore, we can recommended this corrective exercise program to improve the quality of life and their satisfaction in the elderly.

\section{* Corresponding Author:}




\section{Extended Abstract}

\section{Objectives}

H

yperkyphosis in older adults generates severe complications such as limited physical function, balance impairment and reduced quality of life [1-5]. These can lead to falls, frequent fractures, and severe psychiatric complications [6-8]. However, limited studies have investigated the effect of exercises as an intervention for hyperkyphosis on physical function, balance and the quality of life among the elderly, especially elderly men. In most studies, only the effect of corrective training on the severity of hyperkyphosis has been evaluated [9-13]. Thus, the impact of training programs on the complications of hyperkyphosis has received little attention. Severe complications associated with hyperkyphosis have many negative consequences. Therefore, this study aimed to assess the effect of kyphosis corrective exercises on physical function, postural control, and the quality of life in elderly men with hyperkyphosis.

\section{Methods and Materials}

The study participants were 24 elderly men with hyperkyphosis (angle $>50^{\circ}$ ). They were randomly divided into test (Mean \pm SD age $=68.75 \pm 2.67$ years; $M e a n \pm S D$ height=173.66 $\pm 3.75 \mathrm{~cm}$; Mean \pm SD weight $=73.5 \pm 4.75 \mathrm{~kg}$ ), and control (Mean \pm SD age $=69.50 \pm 2.64$ years; Mean \pm SD height $=174.41 \pm 7.78 \mathrm{~cm} ;$ Mean \pm SD weight $=75.41 \pm 5.21 \mathrm{~kg}$ ) groups, each with 12 samples. Inclusion criteria were 65-74 years of age; hyperkyphosis $>50^{\circ}$; physicians' permission to participate in the training program; ability to stand for at least 1 minute and walk 10 meters or more independently or with a conventional cane; no consumption of sedatives or any particular medication affects postural control; the lack of any uncontrollable neurological, respiratory, vascular, metabolic and musculoskeletal diseases; and no serious hearing or visual impairment $[5,24]$.

Exclusion criteria were histories of depression, anxiety or other mental disorders; history of severe articular deformities in the lower extremities and trunk; history of joint replacement in lower extremities; history of balance disorders and vertigo; history of severe pain in the lower extremities and trunk; and receiving physiotherapy program recently or during study [5, 24]. Samples in the test group received a 12-week kyphosis corrective exercises program for 3 sessions per week. Exercises included spinal mobility, occipital muscle stretch, pectoral (chest) muscle stretch, strengthening cervical flexor muscle, and strengthening retractor muscles of the shoulder.

The thoracic kyphosis angle was measured using a flexible ruler at a distance between $T_{2}$ and $T_{12}$ spinous processes. Physical function was examined by 6-min walk test; static balance by sharpened Romberg test (eyes closed and eyes open), and the quality of life by 36-item Short Form Health Survey (SF-36). For analyzing the collected data, Analysis of Covariance (ANCOVA) was performed considering a significance level of $\mathrm{P}<0.05$.

\section{Results}

ANCOVA results suggest that the kyphosis corrective exercises had a significant effect on the quality of life of samples $(\mathrm{F}=6.94, \mathrm{P}=0.04)$. Within group evaluation of

Table 1. Mean and SD of study variables and ANCOVA results before and after the intervention

\begin{tabular}{|c|c|c|c|c|c|}
\hline \multirow{2}{*}{ Variables } & \multirow{2}{*}{ Group } & \multicolumn{2}{|c|}{ Mean $\pm S D$} & \multirow{2}{*}{ Changes (95\% Cl) } & \multirow{2}{*}{ ANCOVA } \\
\hline & & Pre-test & Post-test & & \\
\hline \multirow{2}{*}{ Static balance with eyes open (s) } & Test & $32.7 \pm 3.4$ & $37.4 \pm 4.5$ & $2.33(0.04-4.7)$ & \multirow{2}{*}{$\mathrm{P}=0.02^{* *}$} \\
\hline & Control & $33.3 \pm 3.9$ & $33.7 \pm 4.7$ & $0.33(-1.8-2.5)$ & \\
\hline \multirow{2}{*}{ Static balance with eyes closed (s) } & Test & $10.5 \pm 2.3$ & $12.4 \pm 3.4$ & $0.6(-0.5-1.7)$ & \multirow{2}{*}{$P=0.08$} \\
\hline & Control & $11.4 \pm 2.2$ & $9.8 \pm 3.4$ & $-1.7(-4.7-1.31)$ & \\
\hline \multirow{2}{*}{ Physical function (m) } & Test & $487.7 \pm 33.8$ & $538.9 \pm 32.4^{*}$ & $19.2(13.4-24.8)$ & \multirow{2}{*}{$\mathrm{P}=0.001^{* *}$} \\
\hline & Control & $515.1 \pm 60.6$ & $513.8 \pm 58.5$ & $-1.3(-4.8-2.3)$ & \\
\hline \multirow{2}{*}{ Kyphotic angle (degree) } & Test & $59.33 \pm 5.3$ & $51.83 \pm 4.5$ & $7.50(3.33-11.66)$ & \multirow{2}{*}{$\mathrm{P}=0.005^{* *}$} \\
\hline & Control & $57.66 \pm 2.9$ & $55.70 \pm 6.1$ & $1.95(-2.04-6.04)$ & \\
\hline
\end{tabular}

${ }^{*} \mathrm{P}<0.01 ;{ }^{* *} \mathrm{P}<0.05$ for within group comparisons

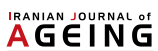


scores by Independent $t$ test revealed that the mean scores of the subscales of bodily pain $(\mathrm{t}=2.01, \mathrm{P}=0.03)$, physical function $(\mathrm{t}=1.89, \mathrm{P}=0.04)$, physical limitation $(\mathrm{t}=2.3$, $\mathrm{P}=0.02)$, general health $(\mathrm{t}=1.93, \mathrm{P}=0.04)$, and mental health $(\mathrm{t}=4.96, \mathrm{P}=0.001)$, improved significantly after the intervention, compared to the pretest scores. However, posttest mean scores of vitality $(\mathrm{t}=0.16, \mathrm{P}=0.87)$, social role $(\mathrm{t}=0.43, \mathrm{P}=0.67)$, and mental limitation $(\mathrm{t}=1.37$, $\mathrm{P}=0.18$ ) showed no significant differences with their pretest mean scores.

ANCOVA results demonstrated that kyphosis exercises had a significant effect on balance, walking, and physical function among samples with hyperkyphosis $>50^{\circ}(\mathrm{P}<0.05)$. Within group comparison results indicated that, in the test group, the mean score of static balance with eyes open $(\mathrm{t}=2.9$, $\mathrm{P}=0.009)$, and physical function ( $\mathrm{t}=3.8, \mathrm{P}=0.001)$ improved significantly after the intervention. However, the mean score of static balance with eyes closed $(\mathrm{t}=1.37, \mathrm{P}=0.018)$ showed no significant improvement. Moreover, kyphosis exercises significantly affected the kyphotic angle in the samples $(\mathrm{P}<0.05)$. With respect to this effect, within group comparison results revealed that the mean score of kyphotic angle in both groups significantly reduced after the intervention $(\mathrm{t}=3.74, \mathrm{P}=0.001)$ (Table 1$)$.

\section{Conclusion}

Kyphosis exercises including stretching exercises, strengthening exercises and spinal movements could improve postural control, physical function, and the quality of life in elderly people with hyperkyphosis $>50^{\circ}$. Improving these factors can be effective in reducing the financial burden of diseases and ageing-related problems, and can provide a basis for life satisfaction in the elderly. Therefore, we recommend this intervention program in order to improve physical function, postural control, and the quality of life in the elderly with hyperkyphosis. However, due to some study limitations, more comprehensive research is required to confirm the obtained results.

\section{Ethical Considerations}

\section{Compliance with ethical guidelines}

All ethical principles were considered in this article. The participants were informed about the purpose of the research and its implementation stages; they were also assured about the confidentiality of their information; Moreover, They were allowed to leave the study whenever they wish, and if desired, the results of the research would be available to them.

\section{Funding}

This research did not receive any specific grant from funding agencies in the public, commercial, or not-forprofit sectors.

\section{Authors contributions}

Conceptualization: Aynollah Naderi and Shahabeddin Bagheri; Draft preparation: Fatemeh Shaabani, Aynollah Naderi, and Shahabeddin Bagheri; Investigation and resources: Fatemeh Shaabani and Mohammad Hossain Rezvani; Editing: Mohammad Hossain Rezvani and Aynollah Naderi.

\section{Conflict of interest}

The authors declared no conflict of interest.

\section{Acknowledgements}

Authors would like to thank the authorities of Welfare Organization and the elderly care centers in of Hamedan For their valuable cooperation. 


\title{
تأثير يك دوره تمرينات اصلاحى كايفوز بر عملكرد جسمانى، تعادل و كيفيت زندكَ مردان سالمند مبتلابه هايير كايفوز
}

\author{
• عينالله نادرى' •، محمدحسين رضوانى'، فاطمه شعبانى' •، شهابالدين باقرى' \\ ا- كروه علوم زيستى ورزش، دانشكده تربيت بدنى و علوم ورزشى، دانشكاه صنعتى شاهرود، شاهرود، ايران.

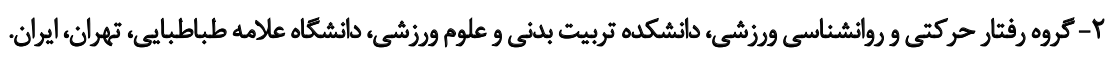

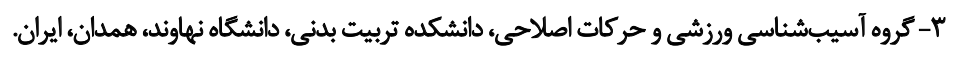

\section{حك}

الهداف در اين تهميق سعى بر آن بود ثأثير يك دوره برنامه اصلاحى كايفوز روى عملكرد جسمانى، تعادل و كيفيث زندكى مردان سالمند مبثلابه هاييركايفوز بررسى شود.

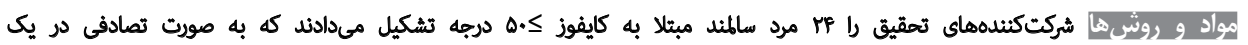
كروه rانفرى آزمايش (با ميانكين سنى سنى St/F/AF

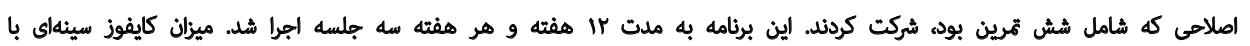

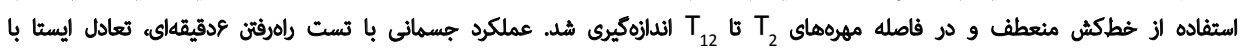

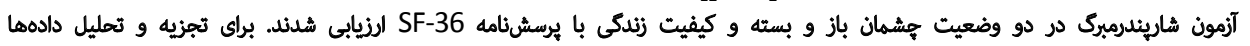

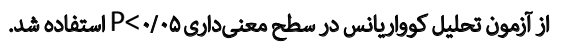

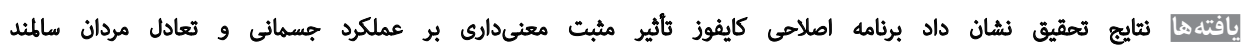

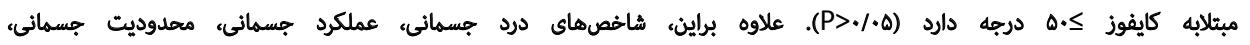

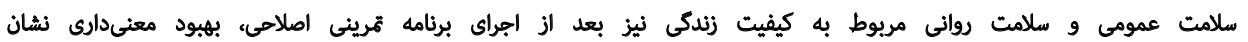

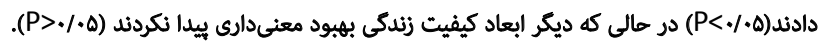

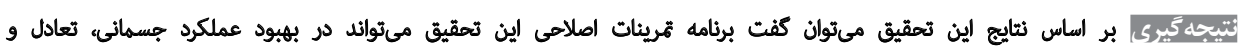

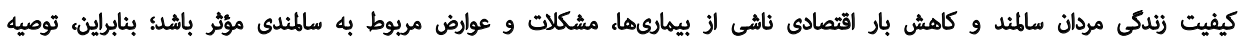

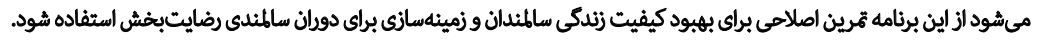

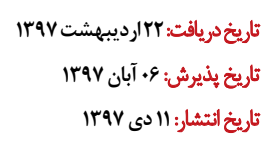

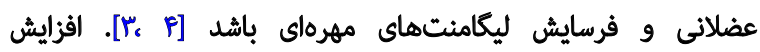

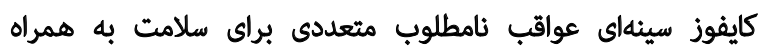

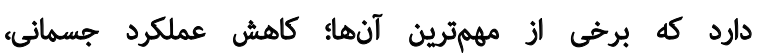

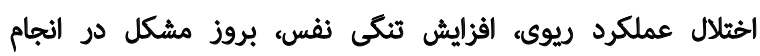

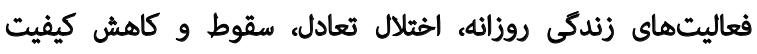

برخى محققان بر اين باورند كه محدوديث انجام فعاليتهاي

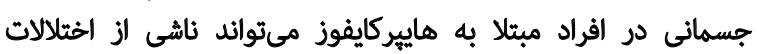

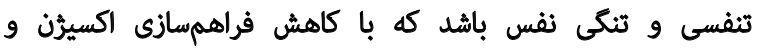

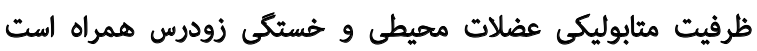

زندكى هم:ن-V]

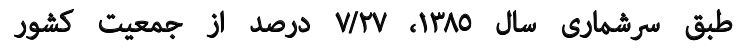

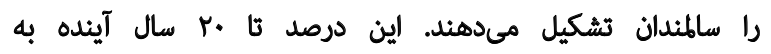

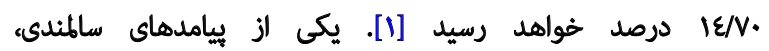

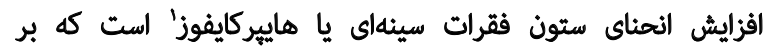

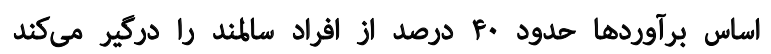

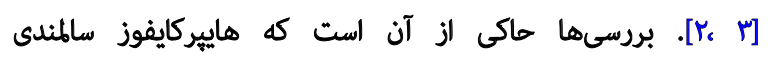
مىتواند ناشى از افزايش سن، شكستكى فشارى مهرهها، اختثلال

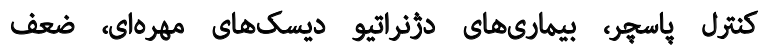

1. Hyperkyphosis

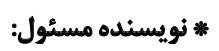

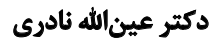

نشانى: ايران، شاهرود، دانشكاه صنعتى شاهرود، دانشكده تربيت بدنى و علوم ورزشى، كروه علوم زيستى ورزش.

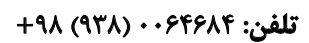
بست الكترونيكى: ay.naderi@yahoo.com 
عمقى ستون فقرات، مشاهده شد طول قد، قدرت اكستشن ستون

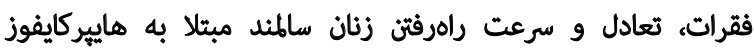

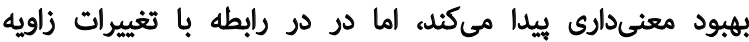
كايفوز نتيجهاى كزارش نشده است [0]

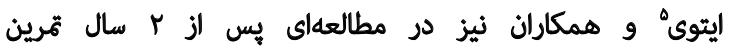

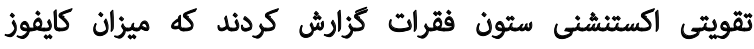

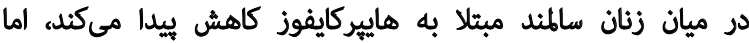

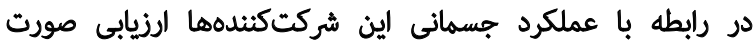

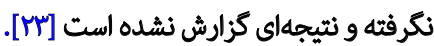

همانطور كه بيان شده هاييركايفوز در سالمندان عوارض كمان

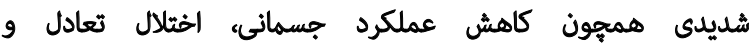

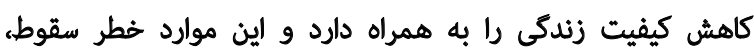

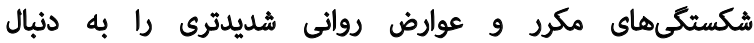

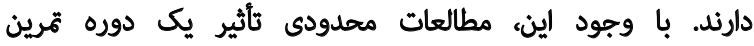
اصلاحى هاييركايفوز را بر عملكرد جسمانى، تعادل و و كيفيت

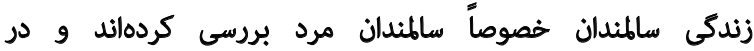

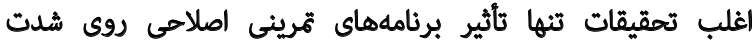

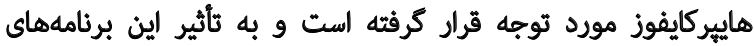
تمرينى روى عوارض ناشى از هاييركايفوز توجه كمى شده است بنابراين، با توجه به شدت عوارض همراه با هاييركايفوز و دوارئ

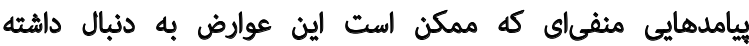

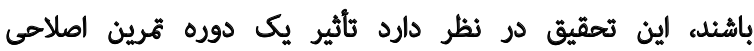

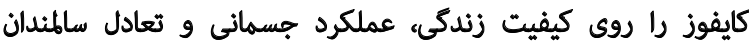

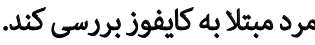
روش مطالعه

اين تحقيق در زمره تحقيقات نيمهتجربى قرار مىكيرد كه

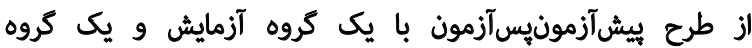

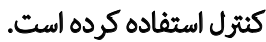

$$
\text { جامعه و نموثه آمارى }
$$

اين تهقيق از نوع تهقيقات نيمهتجربى است كه در

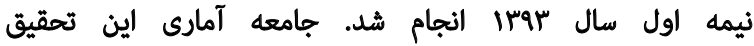

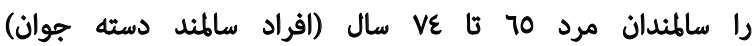

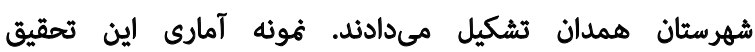

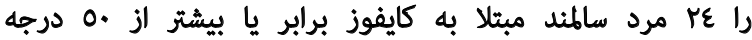

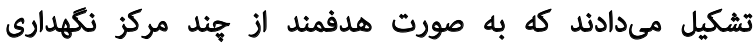

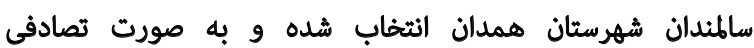
ساده در دو كروه آزمايش (تمرينات اصلاحى كايفوز) با ميانكين سنى SUNDET/TV كثترل با ميانكين سنى على
[م

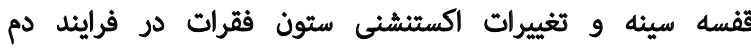

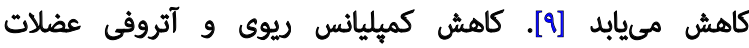

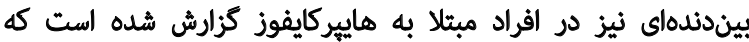

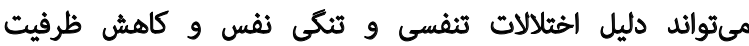
عملكرد جسمانى اين افراد باشد [ل| ، [1].

در سالمندان مبتلا به هاييركايفوز الزدستدادن تعادل عامل

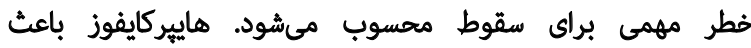

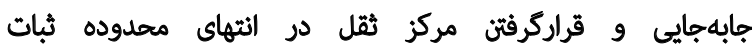

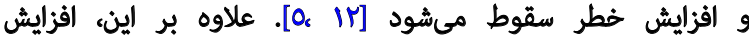

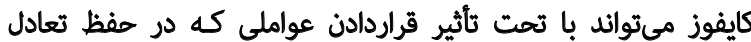

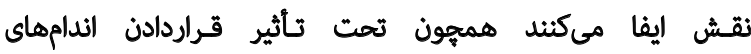

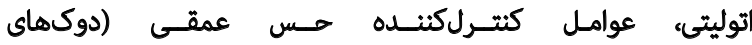

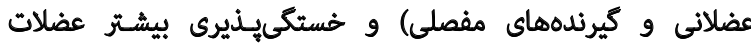

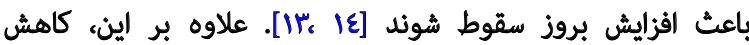

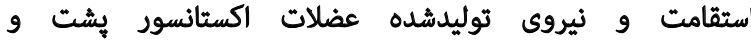

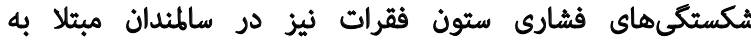

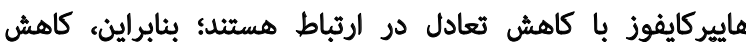

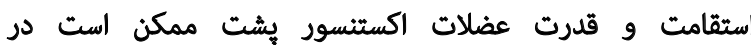
بروز افزايش كيفوز و اغتثلال عملكرد تعادل سهيم باشئد [10]. هاييركايفوز به صورت مستقيم (از طريق زيباشناختى) و ونانيق

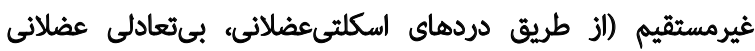

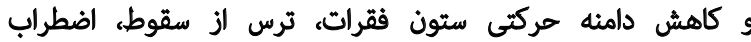

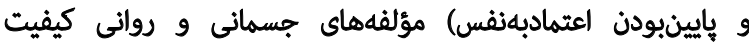

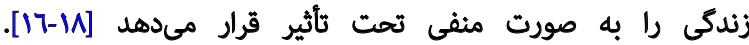

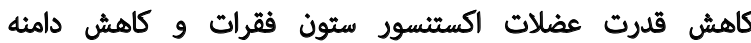

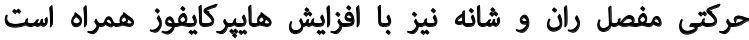

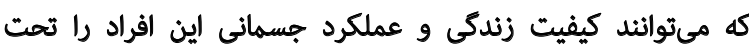

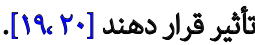

هاييركايفوز از طريق روشهاى درمانىاصلاحى متفاوتى تحت

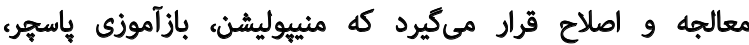

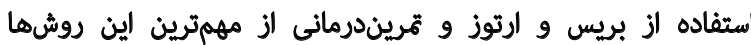

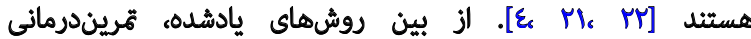

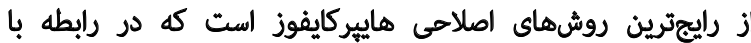

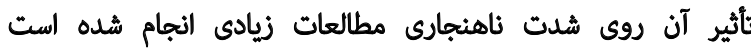

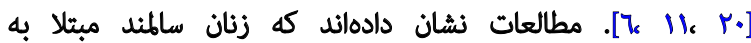
هاييركايفوز بعد از IT هفته تمرينات اصلاحى يوكا در آزمونهاى

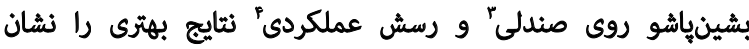

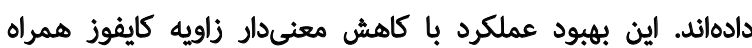

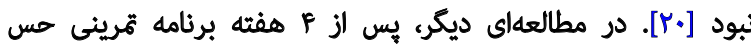

2. Expansion

3. Chair stand

4. Functional reach 


\section{اندازهتيرى زاويه كايفوز}

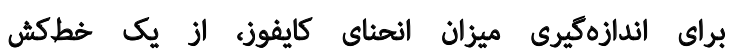

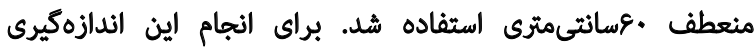

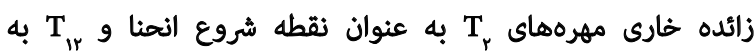

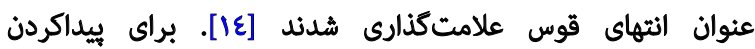

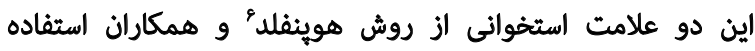

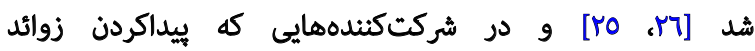

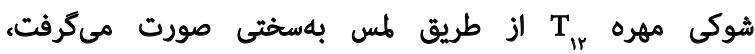

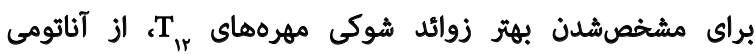

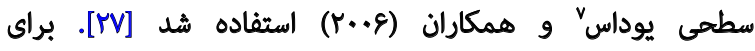
محاسبه زاويه كايفوز سينهاي، اطلاعات بهدست همآمده دران مر معادله

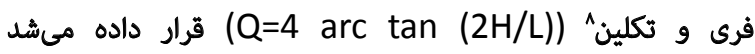

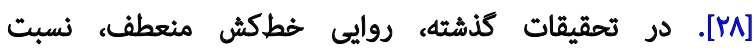

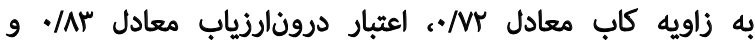

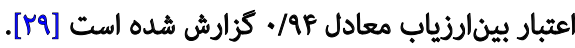

\section{كيفيت زندمّى}

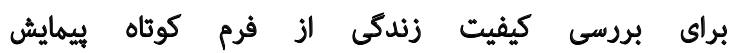

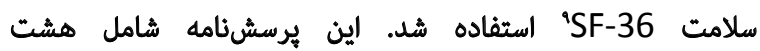

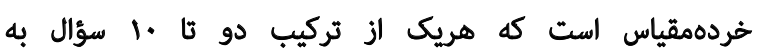

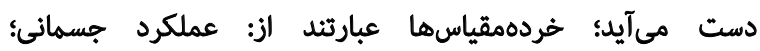

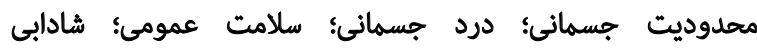

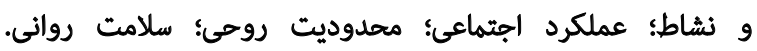

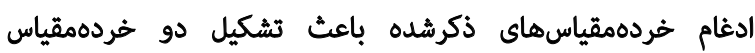

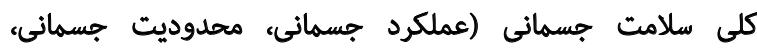

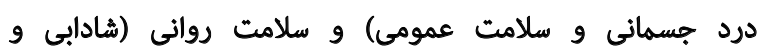

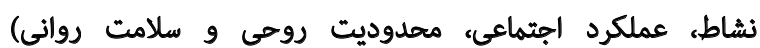

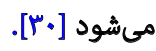

اين يرسشنامه را ميتوان هم با يك فرد و هم بم به كمك

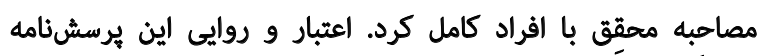

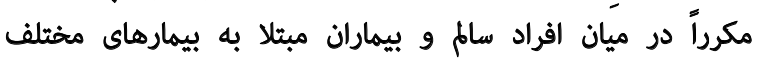
ارزيابى شده است. برازير و همكاران در مطالعهاي اعتبار نسخه

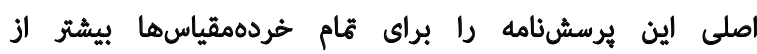

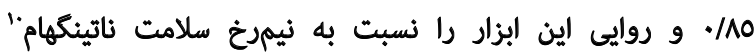

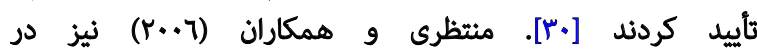

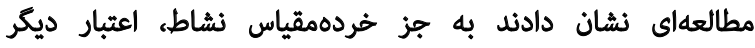

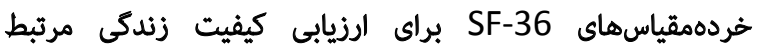

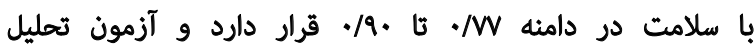

6. Hoppenfeld

7. Youdas

8. Frey \& Tecklin

9. Short Form Health Survey

10. Nottingham health profile

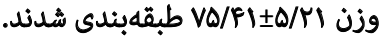

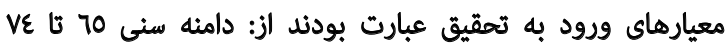

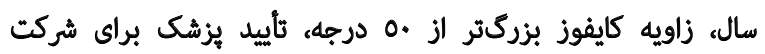

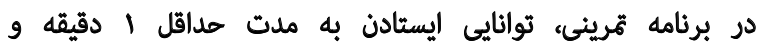

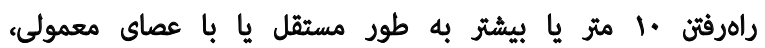

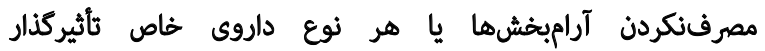

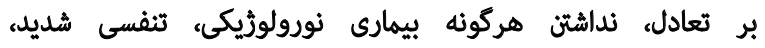

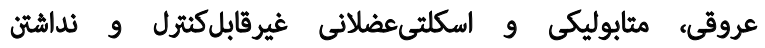

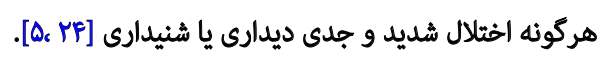

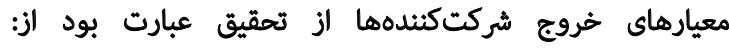

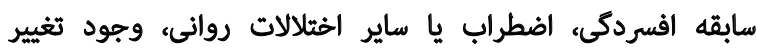

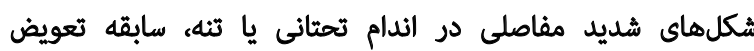

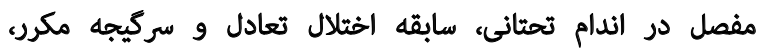

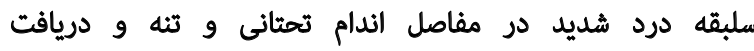

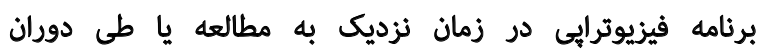

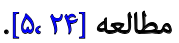

شيوه انتخاب شركتكنثهها به اين شكل بود كه محقق در

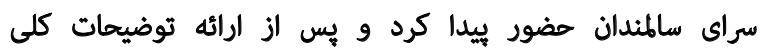
در رابطه با موضوع مطالعه و كسب رضايث شفاهى از سالمندان،

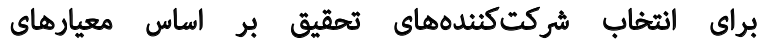

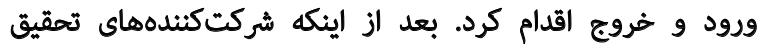

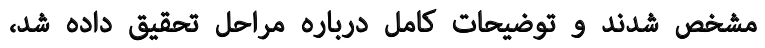

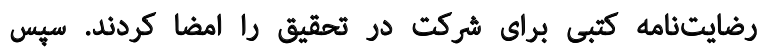

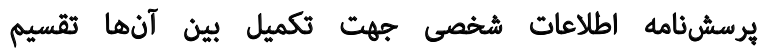

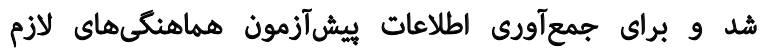

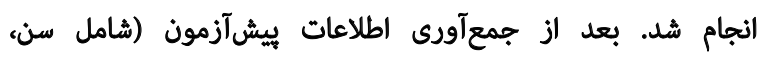

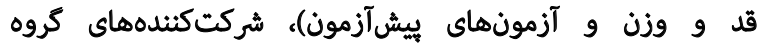

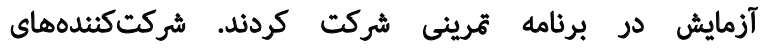

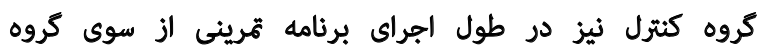

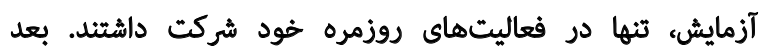

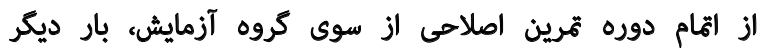
اندازهيرىهاى صورت كرفته در بيش آزمون تكرار شيد. دو نفر از شركتكنندهاي كروه آزمايش به دليل مشاركتنكردن

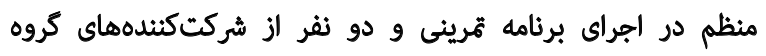

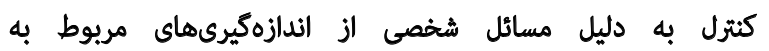
يسآزمون حذف شدنل. در اين تحقيق شركت شنندههاى كروه

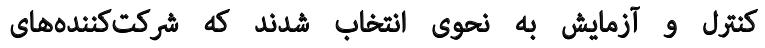

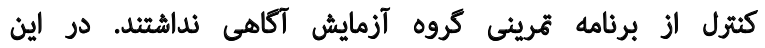

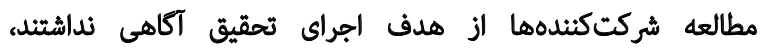

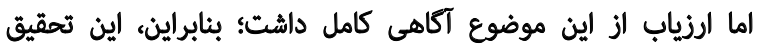

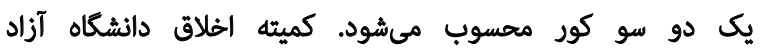
اسلامى واحد همدان اين بُؤوهش را تأييد كرده است. 

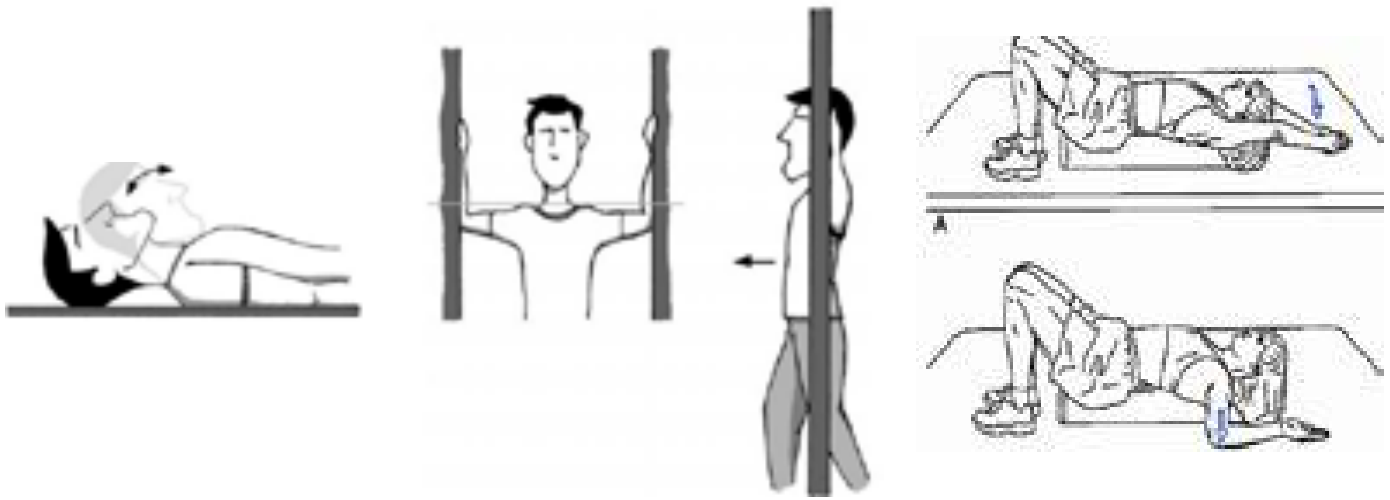

تصوير ج؛ تقويت عضلات فلكسور گردن

تصوير ب؛ كشش عضلات سينهاى

تصوير الف؛ تحركبخشى ستون فقرات

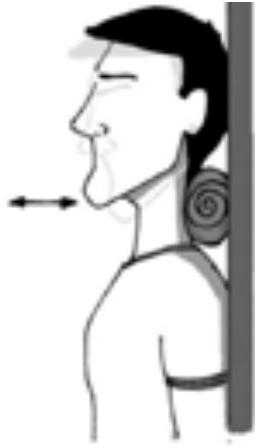

تصوير و؛ كشش عضلات پسسرى (جين

تاكى)
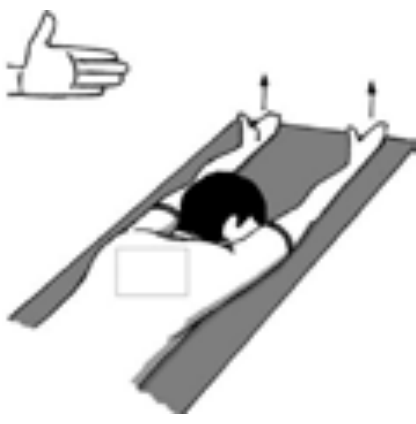

تصويره؛ تقويت عضلات ريتراكتور كتف

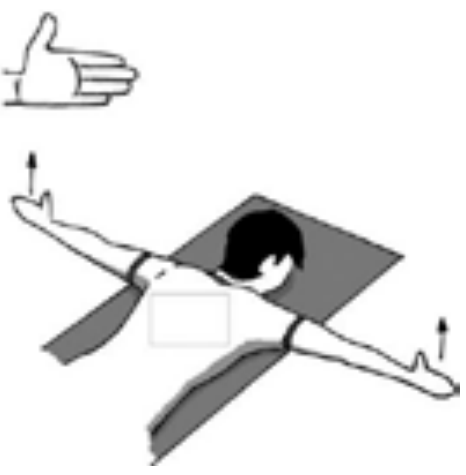

تصوير د؛ تقويت عضلات ريتراكتور كتف

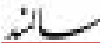

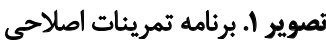

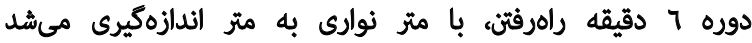

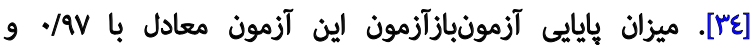

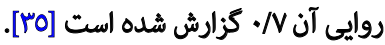

$$
\text { برنامه تمريني }
$$

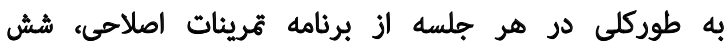

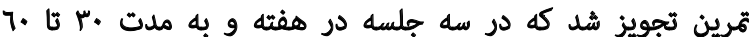
دقيقه اجرا مىشد. در ابتدا و انتهاى هر جلسه ثمرينى نيز هر

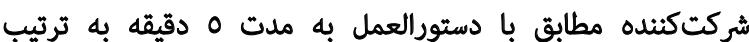

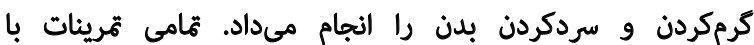

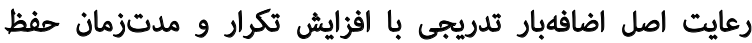

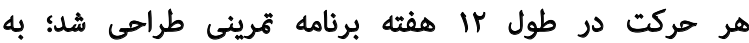

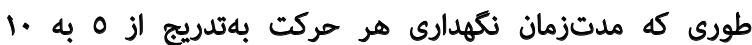
ثانيه و تعداد تكرار هر ست از 7 به ·. حركت افزايش يافت.

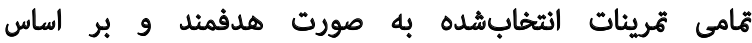

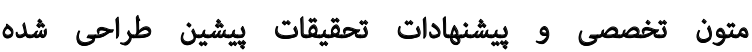

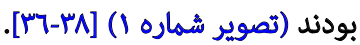

عاملى ثيز دو خردهمقياس اصلى را مشخص كرده و روايى

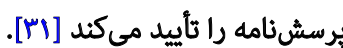

تعادل ايسئا

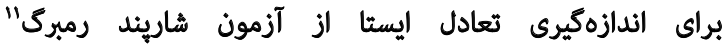

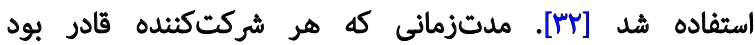

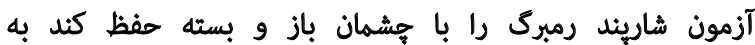

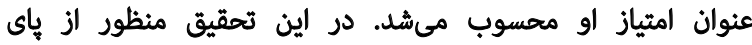

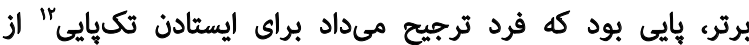

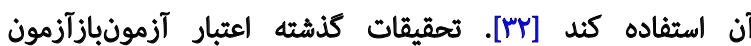

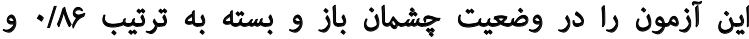

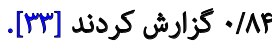

$$
\text { عملكرد جسمانى }
$$

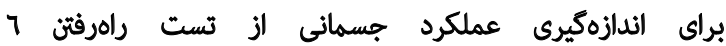

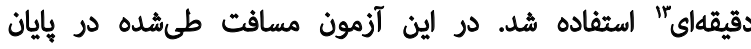

11. Sharpened Romberg Test

12. One leg standing

13. 6-Minute Walk Test 


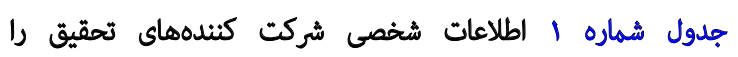

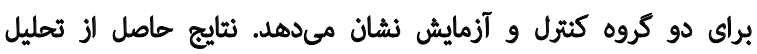

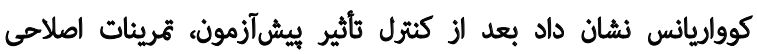

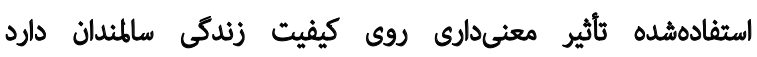

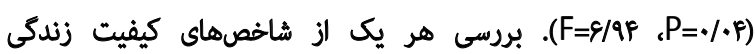

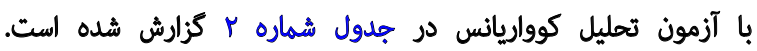

اطلاعات كردآورىشده بر اساس سؤالات تحقيق و با استفاده از

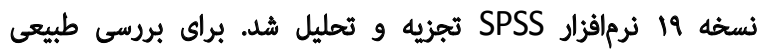

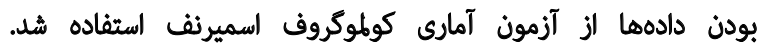

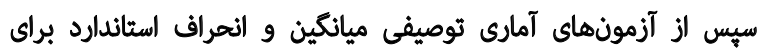

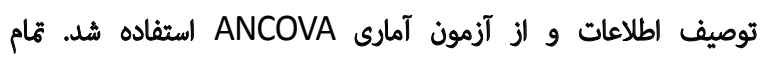

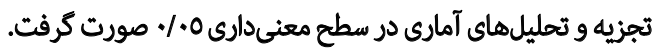

جدول ا. مشخصات فردى شركتكثندهاي تحقيق

\begin{tabular}{|c|c|c|}
\hline \multicolumn{2}{|c|}{ كروهها } & \multirow{2}{*}{ مثغيرها } \\
\hline كتترل & آزمايش & \\
\hline$s q / \Delta+ \pm T / q F$ & $\& N Y \Delta_{ \pm} r / F \&$ & سن (سال) \\
\hline$V \Delta / T_{ \pm} I_{ \pm} \Delta / T I$ & $V / \Delta_{ \pm} F / V \Delta$ & ونن (كيلوكرم) \\
\hline $\mid V f / F_{ \pm} V / V \Lambda$ & $\mid r / \varepsilon_{ \pm} r / V \Delta$ & قد (سانتىمتر) \\
\hline
\end{tabular}

2

جدول r. نتايج آزمون ANCOVA مربوط به مقايسه شاخصهاى كيفيت زندكى سالمندان

\begin{tabular}{|c|c|c|c|c|c|}
\hline \multirow{2}{*}{ ANCOVA } & \multirow{2}{*}{ تغييرات (ه) / Cl) } & \multicolumn{2}{|c|}{ ميانكين土 انحراف معيار } & \multirow{2}{*}{ كروه } & \multirow{2}{*}{ متغيرها } \\
\hline & & يس أزمون & يميش آزمون & & \\
\hline \multirow{2}{*}{$\mathrm{P}=* / \cdot r_{* * *}$} & $\Delta / \Delta(\cdot / r-V / \Lambda)$ & $9 / / \perp_{ \pm} \Delta / 9$ & $\Lambda \otimes / \varepsilon_{ \pm} V / F$ & آزمايش & \multirow{2}{*}{ درد جسمائي } \\
\hline & $\cdot / \Delta(-1 / \Delta-Y / \Delta)$ & $N ه / \Delta_{ \pm} F / f$ & $1 \otimes / \bigwedge_{ \pm} \& / 1$ & كتترل & \\
\hline \multirow{2}{*}{$\mathrm{P}=* / \cdot r^{* *}$} & S/N(-r/r-NA) & $N E / I_{ \pm} V / q$ & $V q / r_{ \pm} q / V$ & أزمايش & \multirow{2}{*}{ عملكردجسمائى } \\
\hline &.$/ V(-g / \Delta-V / A)$ & $1 \cdot 1+1 \pm 9 / r$ & $r q / \Delta_{ \pm} \varepsilon / T$ & كنترل & \\
\hline \multirow{2}{*}{$\mathrm{P}=* 1++\varphi$} & $V / I V(-Y / \Lambda-V / \Lambda)$ & $W / N_{ \pm} \Delta / V$ & $q 1 / 1 \varepsilon_{ \pm} N q$ & أزمايش & \multirow{2}{*}{ محلدوديت جسماثيى } \\
\hline & $-r / r(-r / Q-V / \Delta)$ & $9 . / 8 \pm p / q$ & $9 r / q_{ \pm} \& / \Delta$ & كنترل & \\
\hline \multirow{2}{*}{$P=\star / \bullet \bullet \Lambda^{* *}$} & $\Delta / \Delta(\cdot / r-s / V)$ & $\Lambda T / \Lambda_{ \pm} V / \mathcal{F}$ & $v \&\left|\varepsilon_{ \pm}\right| \cdot / 1$ & 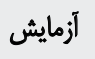 & \multirow{2}{*}{ سلامت عمومى } \\
\hline &. $\mid F(-r / \varepsilon-r / \Lambda)$ & $v \varepsilon / \Delta_{ \pm} \varepsilon / r$ & $V \Delta / q_{ \pm} q / r$ & كنترل & \\
\hline \multirow{2}{*}{$\mathrm{P}=. / e$} &.$/ M T(-1 / F-r / 1)$ & $8 \Delta / \cdot \cdots \pm V / \varepsilon$ & $g / \varepsilon_{ \pm} f / \varepsilon$ & آزآمايش & \multirow{2}{*}{ شادابى و نشاط } \\
\hline &.$/(-g / T-r / 8)$ & $\varepsilon r / r_{ \pm} \varepsilon / q$ & $R / / T_{ \pm} \Delta / V$ & كنترل & \\
\hline \multirow{2}{*}{$P=\varnothing / \Delta$} & $V / \Delta \cdot(-\Delta / V-N A)$ & $9 \Psi / \Lambda_{ \pm} F / \Delta$ & $9 Y / T_{ \pm}+1 \cdot / 1$ & آزمايش & \multirow{2}{*}{ عملكرداجتماعي } \\
\hline & $\cdot / V(-1 / \Gamma-Y / \varepsilon)$ & $91 / \varepsilon_{ \pm} V / T$ & $9) / \Lambda_{ \pm} N{ }^{\mu}$ & كنترل & \\
\hline \multirow{2}{*}{$P=\star / \bullet q$} & $1 / V \Delta(-1 / 1-r / \Delta 1)$ & $q V / \varepsilon_{ \pm} r / T$ & $9 \Delta / \Lambda_{ \pm} r / q$ & أزمايش & \multirow{2}{*}{ مجدوديث روائي } \\
\hline & $1 / 1(-\cdot / 9-F / \Delta)$ & $q V / \cdot \bullet \pm r / 1$ & $9 \Delta / r_{ \pm}+r / 1$ & كنترل & \\
\hline \multirow{2}{*}{$P=\varnothing / . \bullet r$} & $1 \Delta / T^{e}(-r / \Delta-V / \Psi)$ & $M q_{ \pm} V / \Delta$ & $V / \Delta_{ \pm} V / V$ & 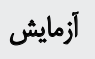 & \multirow{2}{*}{ سلامت روائى } \\
\hline &.$/ 9(-\Delta / r-1 / q)$ & $V \cdot / r_{ \pm} \Delta / 9$ & $V / R \pm E / V$ & كثترل & \\
\hline
\end{tabular}




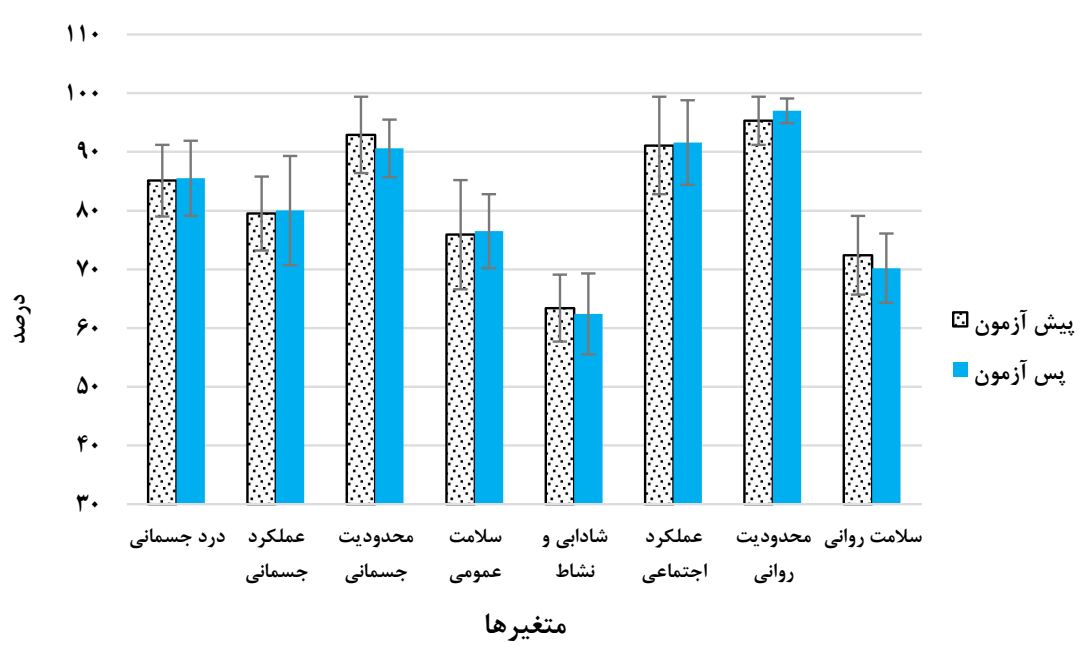

2

ميانكين تعادل، راهرفتن و عملكرد جسمانى شركتكنندههاى

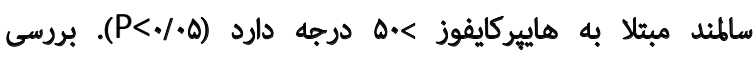

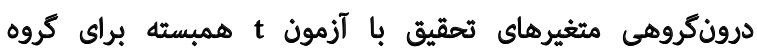

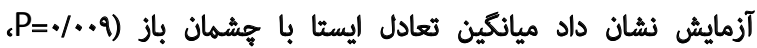
(t=r/9

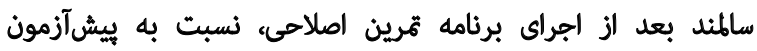

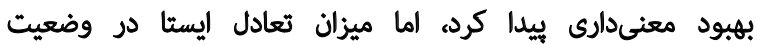

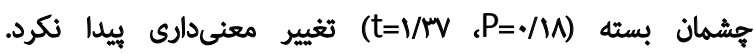

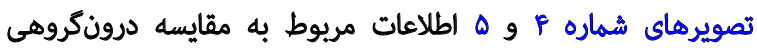

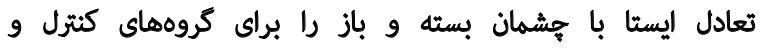

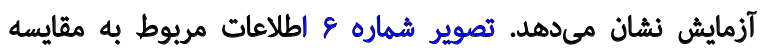
درونكروهى عملكرد جسمانى براى كروههاى كنترل و آنمايش

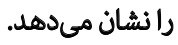

تصوير T. مقايسه درون كروهى خردهمقياس هاى كيفيت زندكى براى كروه كنترل

بررسى درونكروهى يافتههاى تحقيق با آزمون تى همبسته براى كروه

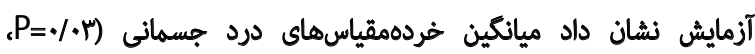
(t=Y/.1

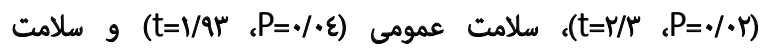

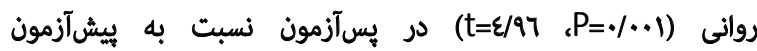

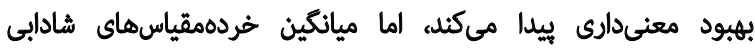

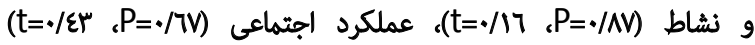

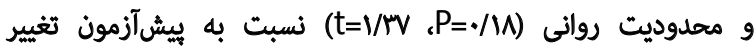

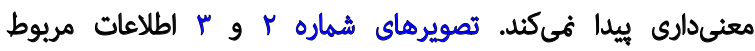

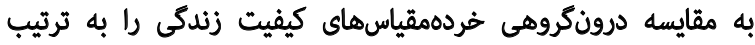
براى كروه هاى كنترل و آزمايش نشان مئدهن دردئ.

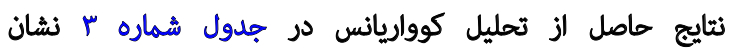

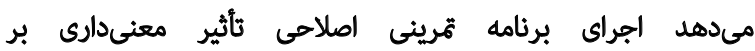

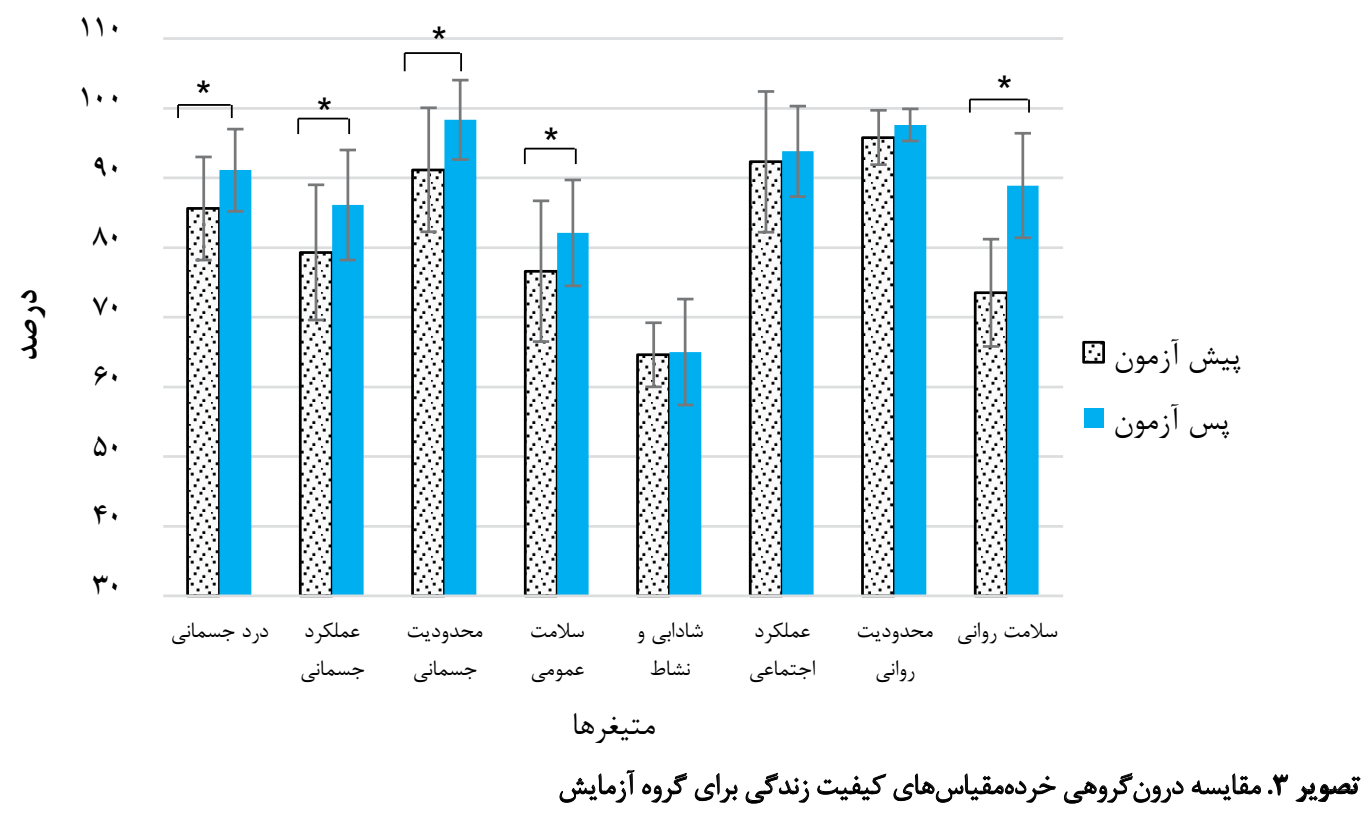


جدول ب. نتايج آزمون ANCOVA مربوط به مقايسه شاخص هاى تعادل، اطمينان حركتى و عملكرد جسمانى

\begin{tabular}{|c|c|c|c|c|c|}
\hline ANCOVA & تغييرات (Cl / (9ه) & 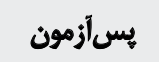 & 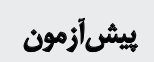 & تمروه & مثغيرها \\
\hline \multirow{2}{*}{$P=+1 \cdot r^{* *}$} & $T / \Psi T(+/+F-F / V)$ & $r V / \Psi_{ \pm} E / \Delta$ & $r r / V_{ \pm} \Psi / \mathcal{C}$ & آزمايش & \multirow{2}{*}{ تعادل ايستا با جشمان باز (ثائيه) } \\
\hline &.$/ M(-1 / A-r / \Delta)$ & $M / V_{ \pm}+N$ & $M / r_{ \pm} H / q$ & كثترل & \\
\hline \multirow{2}{*}{$\mathrm{P}=. / \cdot \wedge$} & $\cdot / 8(-\cdot / \Delta-1 / V)$ & $\mid r / E_{ \pm} r / F$ & $1 \cdot / \Delta_{ \pm} r / T$ & أزمايش & \multirow{2}{*}{ تعادل ايستا با جشمان بسته (ثانيه) } \\
\hline & $-1 / V(-r / V-1 / r 1)$ & $V / \Lambda_{ \pm} r / f$ & $W / F_{ \pm} r / r$ & كتترل & \\
\hline \multirow{2}{*}{$P=\star / . .1 *$} & $19 / T(\mid r / T-r F / \Lambda)$ & 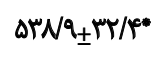 & $P A V / V_{ \pm} \pi T / \Lambda$ & آزمايش & \multirow{2}{*}{ عملكرد جسمانى (متر) } \\
\hline & $-I / r(-Y / A-r / r)$ & $\Delta \| r / \Lambda_{ \pm} \Delta N \Delta$ & $\Delta) \Delta / \Lambda_{ \pm} \varepsilon \cdot / \varepsilon$ & كنترل & \\
\hline
\end{tabular}

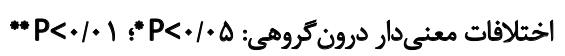

جدول F. نتايج آزمون ANCOVA مربوط به مقايسه بين كروهى زاويه كايفوز بين كروه كنترل و آزمايش

\begin{tabular}{|c|c|c|c|c|c|}
\hline ANCOVA & تغييرات (Cl \% (هه) & يسأزمون & ييش آزمون & كروه & متغيرها \\
\hline \multirow{2}{*}{$P=\star / \circ \Delta \Delta^{\infty * \infty}$} & $V / \Delta \cdot(r / r r-11 / \varepsilon 9)$ & $\Delta V / \lambda r_{ \pm} F / \Delta$ & $\Delta Q / T_{ \pm} \Delta / r$ & آزمايش & \multirow{2}{*}{ زاويه كايقوز (درجه) } \\
\hline & $1 / 9 \Delta(-r / \cdot F-s / \cdot F)$ & $\Delta \Delta / V \cdot \pm \varnothing / 1$ & $\Delta V / \& \varepsilon_{ \pm} r / q$ & كنترل & \\
\hline
\end{tabular}

\section{2}

سالمند مبتلا به هاييركايفوز بود. نتايج اين تحقيق نشان داد

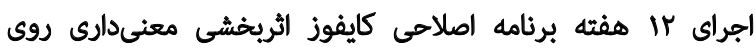

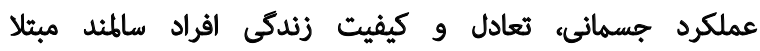

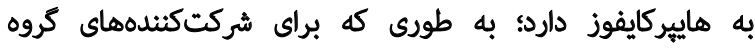

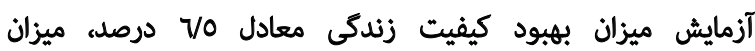

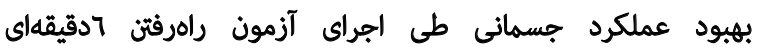

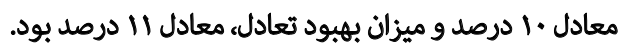

ارزيابى كيفيت زندكى مرتبط با سلامت به عنوان يك

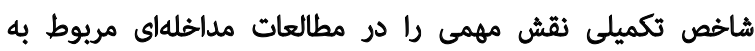

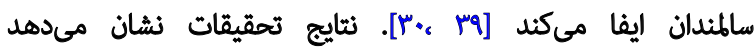

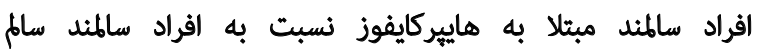

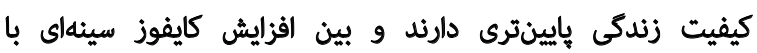
كاهش كيفيث زندكى ارتباط معنى

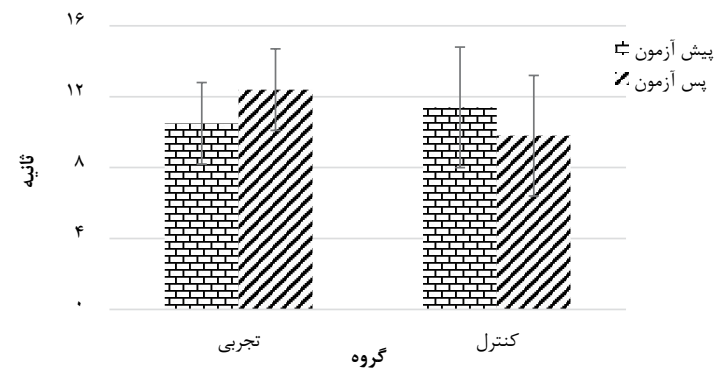

نـ

تصوير هـ مقايسه درون كروهي ثعادل ايسثا با جشمان بسته براي كروه

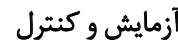

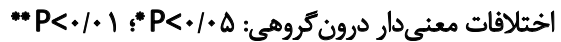

نثايج حاصل الز تحليل كوواريانس در جدول شماره F نشان

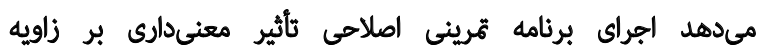

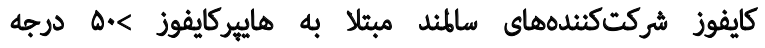

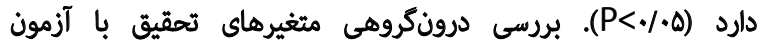

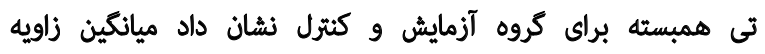

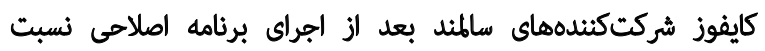

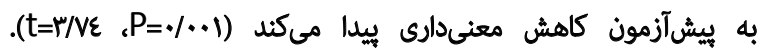

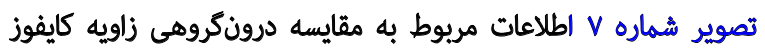
رابراى كروههاى كنتزل و آزمايش نشان مى لهـهد. $\stackrel{\leftrightarrow}{\oplus}$ هدف الز اين تحقيق بررسى تأثير يك دوره تمرينات اصلاحى

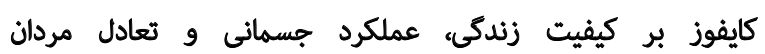
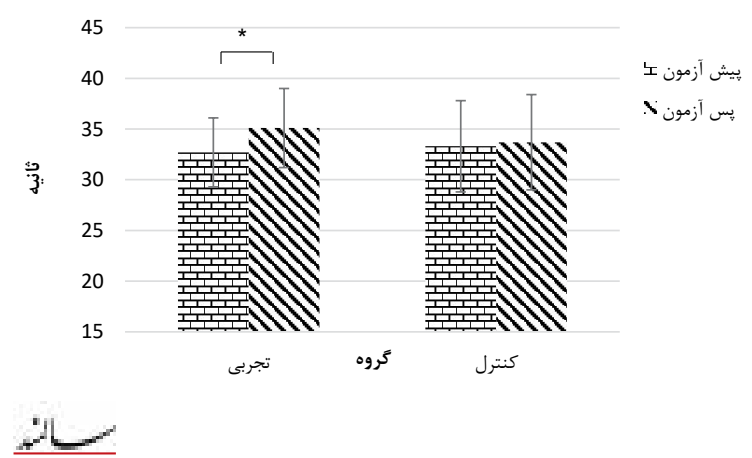

تصوير F. مقايسه درون كروهى تعادل ايستا باجشمان باز براي كروه آزمايش و كنترل 


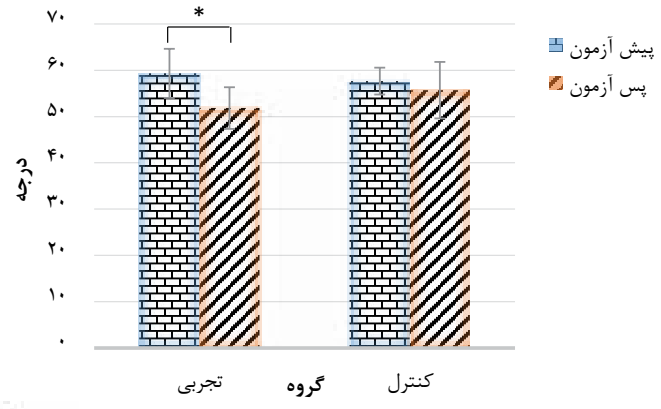

2

تصوير Y. مقايسه درون كروهى زاويه كايفوز مربوط به دو كروه كنترل و آزمايش

دارند. معمولاً در اين آزمون، عملكردى به صورت بالينى براى

ييشبينى سقوط استفاده مى آشود [1]].

نتايج اين تحقيق نشان داد IT هفته برنامه اصلاحى هاييركايفوز

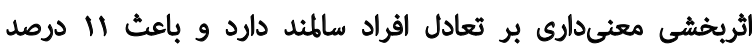

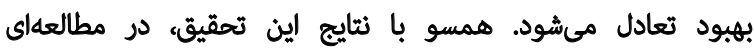

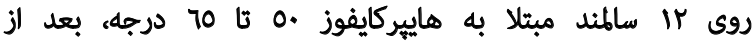

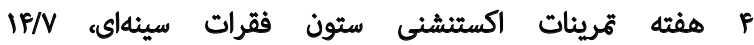

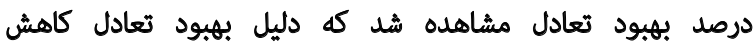

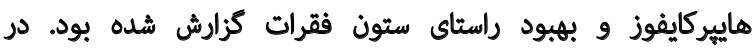

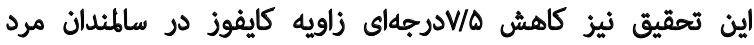

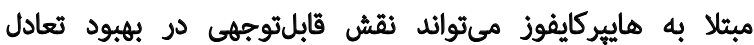

اين افراد داشته باشد [0].

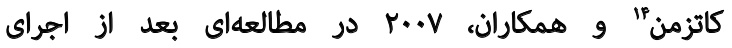

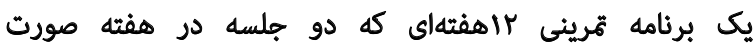

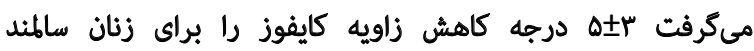

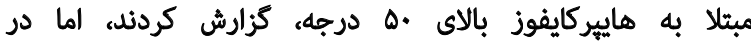

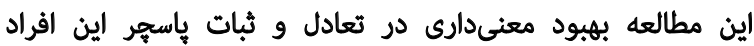

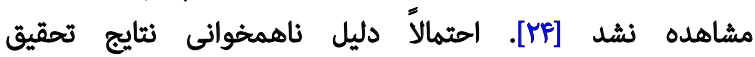

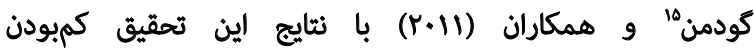

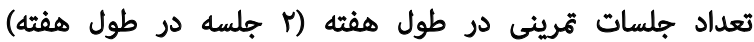

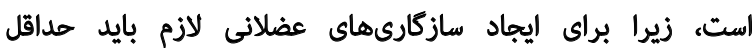

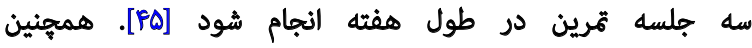

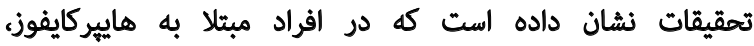

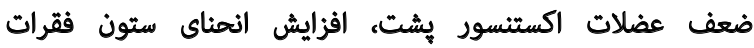

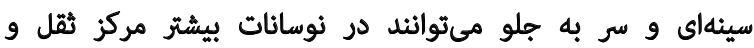

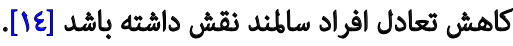

عضلات اكستنسور يشت جزء عضلات ياسجرال ضدجاذبه

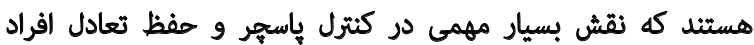

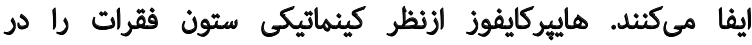

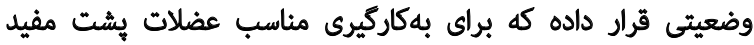

14. Katzman

15. Goodman

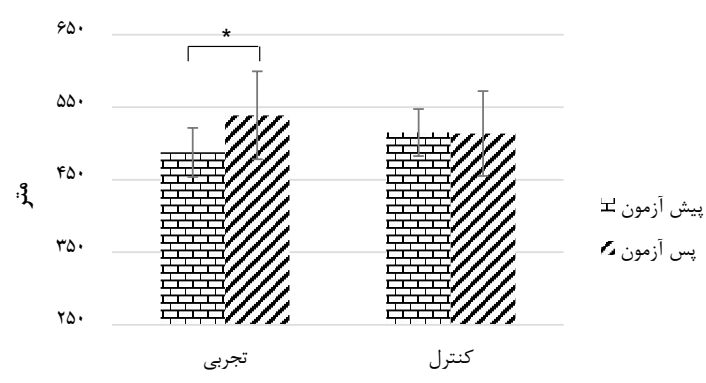

2

تصوير ؤ مقايسه درونكروهى عملكرد جسمانى براى كروه آزمايش و كثترل

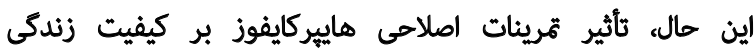

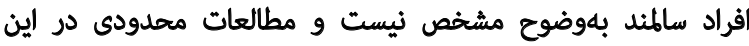

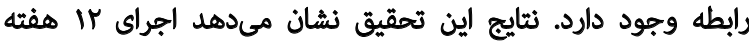

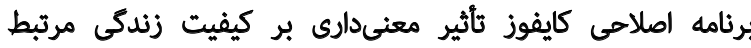
با سلامت سالمندان مبتلا به هاييركايفوز دارد.

همسو با نتايج اين تحقيق، در مطالعهاى نشان داده شده

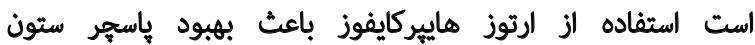

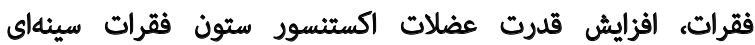

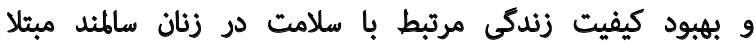

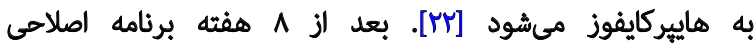

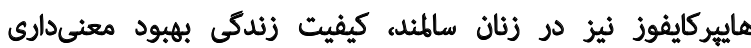

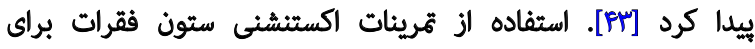

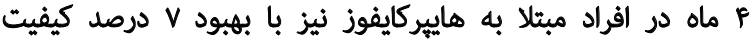

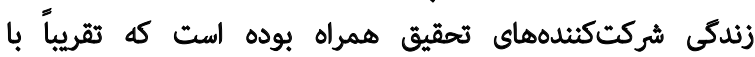

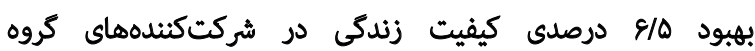
تمرينى اين تحقيق همخوانى دارد.

ثمرينات اصلاحى از طريق افزايش قدرت، انعطافيذيرى و وإئو

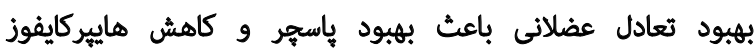

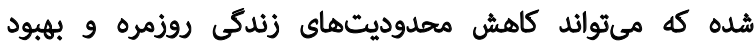

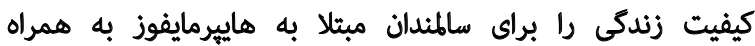

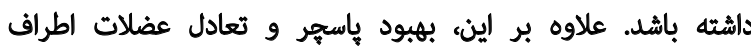

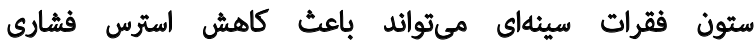

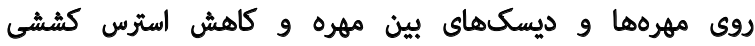

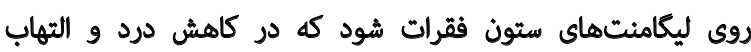

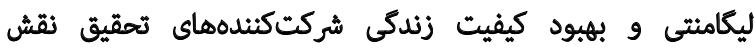

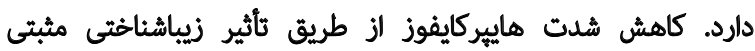
كه دالرد نيز مى تواند درنهايت منجر به كيفيت بهتر زندكى مى إئود. بررسى مطالعات نشان مىدهد افزايش كايفوز در افراد سالمنده

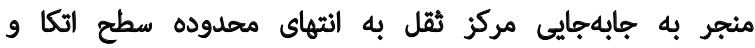

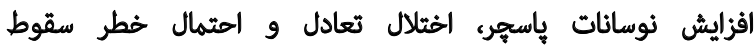

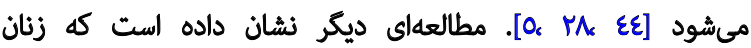

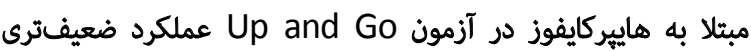


اجراى برنامه اصلاحى كايفوز در اين مطالعه ميتواند از طريق

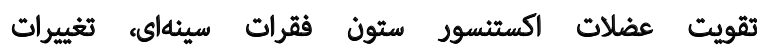

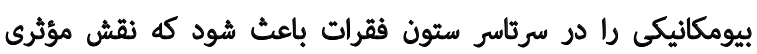

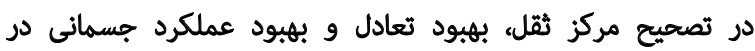

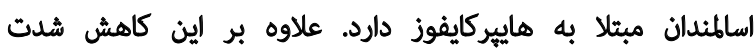
هاييركايفوز ميتواند نقش مؤثرى در توسعهيذيرى قفئه

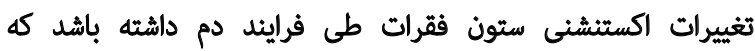

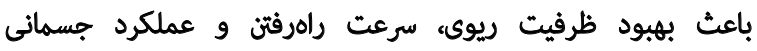

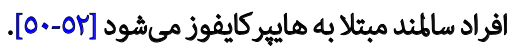

\section{تثيجلكيّى نهايى}

نثايج اين تمقيق نشان داد تمرينات اصلاحى كايفوز شامل:

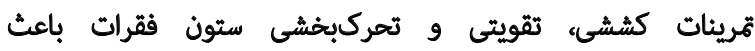
بهبود تعادل، عملكرد جسمانى و كيفيت زندكى مردان سالميند

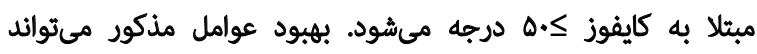

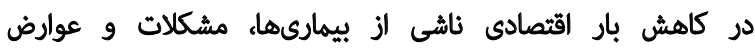

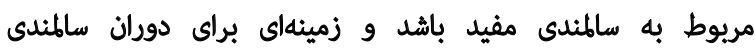

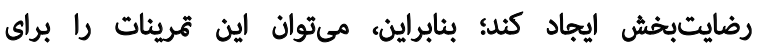

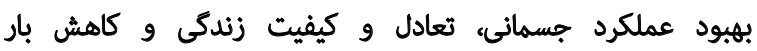

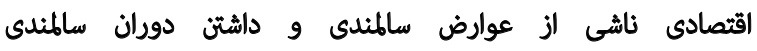

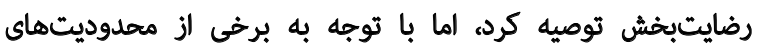

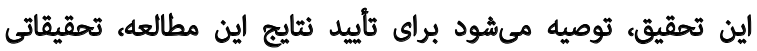
جامعتر النجام شود.

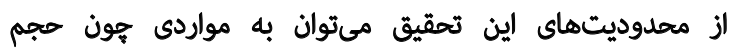

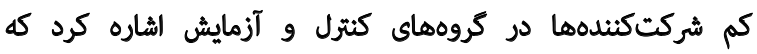

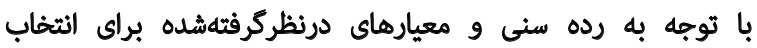

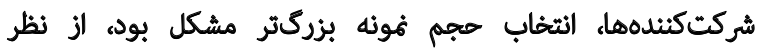

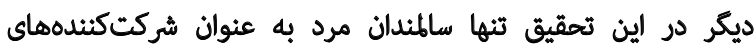
تحقيق انتخاب شدند كه ميتواند باعث تهفي محدوديت تعميميذيرى

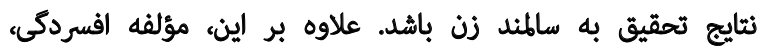

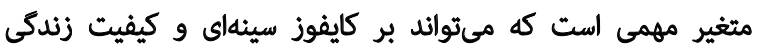

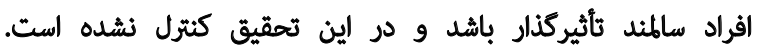

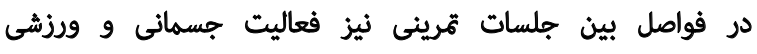

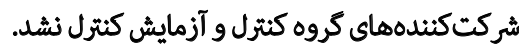

ممكن است در اين فواصل زمانى شركت شنثدها فعاليتهاى

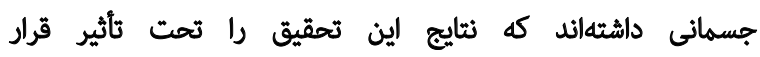

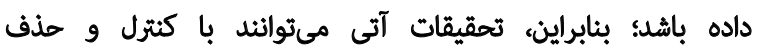

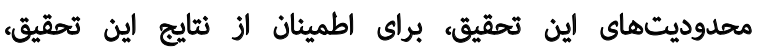

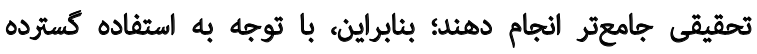

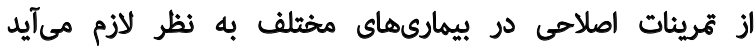

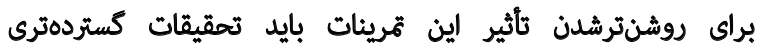

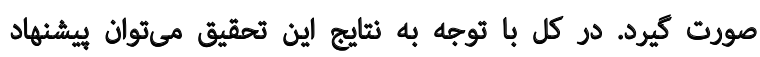

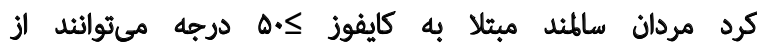

نيست [7ع]. از نظر ديكر به دليل كشش مداوم عضلات اكستنسور

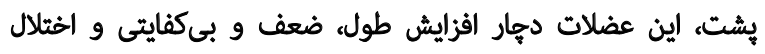

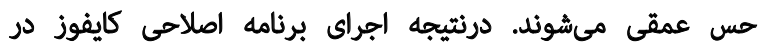

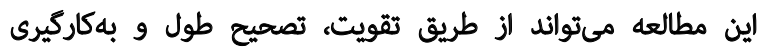

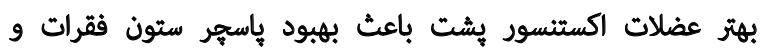
بهبود تعادل افراد مبتلا به هاييركايفوز شود.

علاوه بر اين، هاييركايفوز الز طريق تهت تأثير قراردادن

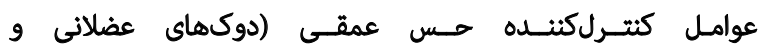

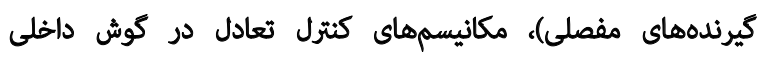

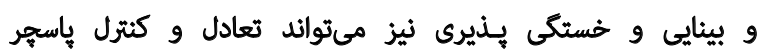

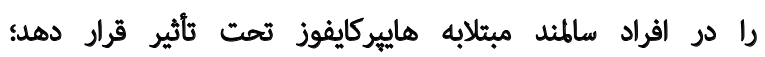

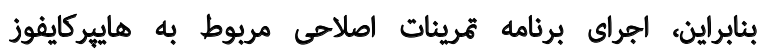

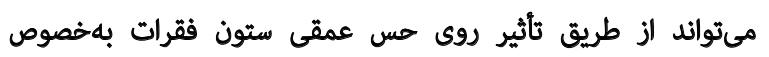

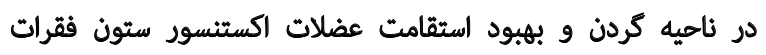
به عنوان كروه عضلات ضدكرانش و اصلاح وضعيت سر و و درنتيجه

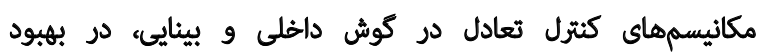
تعادل افر الد سالمند نقش ايفا كند.

مطالعات نشان داده الست افزايش كايفوز با كاهش سرعت

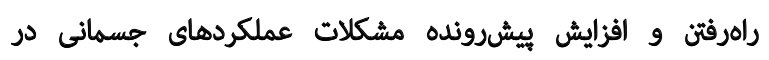

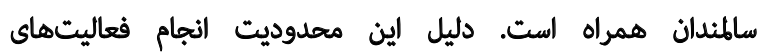

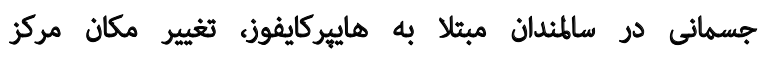

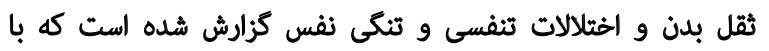

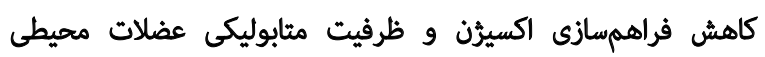

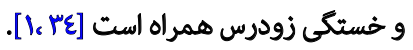

نثايج اين تحقيق نشان داد بعد الز الجراى Ir إن هفته برنامه ثمرينى

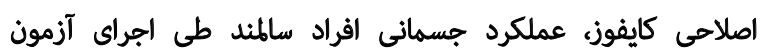

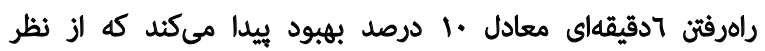

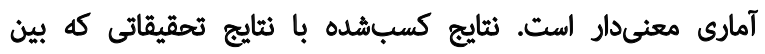

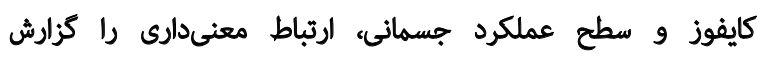

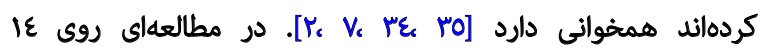

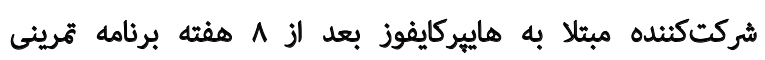

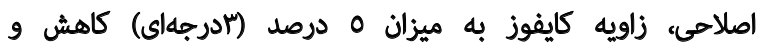

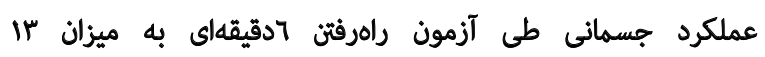

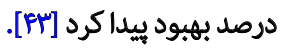

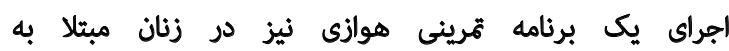

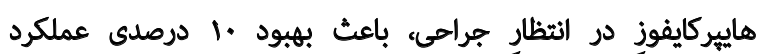

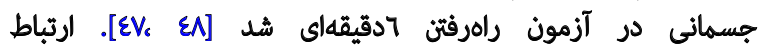

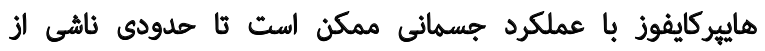

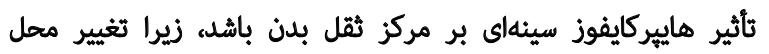

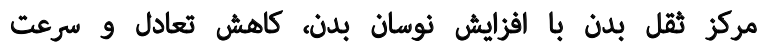

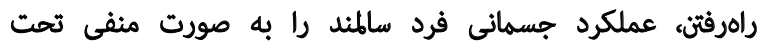

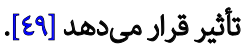


تمرينات اصلاحى كايفوز كه در اين تحقيق ارائه شده است براى

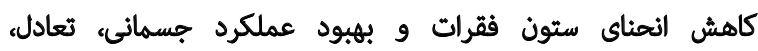
اطمينان حركتى و برخى از مؤلفه هاى كيفيت زئدكى سود بيرند.

ملاحظات اخلاقى

$$
\text { بيروى از اصول اخلاق يثوهش }
$$

همه اصول اخلاقي در اين مقاله رعايت شيده است.

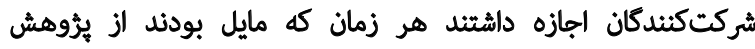

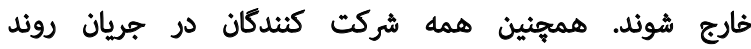

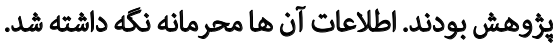

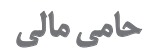
بنابر اظهار نويسندكان، اين مقاله مامى مالى نداشته است.

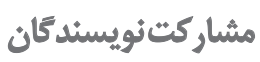

مفهومسازي: عينالله نادرى و شهابالدين باقرى؛ نكارش

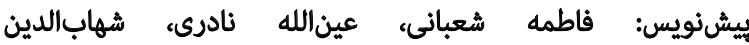
باقرى؛ تحقيق و بررسى منابع: فاطمه شعبانى و محمداحسين

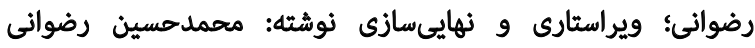
و عينالله نادرى.

$$
\text { تُعار منافع }
$$

بنابر اظهار نويسندكان، اين مقاله تعارض منافع ندارد.

$$
\text { تشكر و قدردانى }
$$

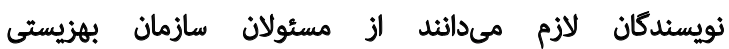

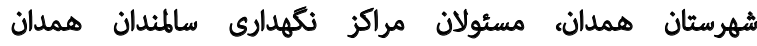
كمال تشكر و قدردانى را داشته باشئد. 


\section{References}

[1] Katzman WB, Vittinghoff E, Kado DM. Age-related hyperkyphosis, independent of spinal osteoporosis, is associated with impaired mobility in older community-dwelling women. Osteoporosis International. 2011; 22(1):85-90. [DOI:10.1007/s00198-010-1265-7]

[2] Kado DM, Huang MH, Karlamangla AS, Barrett-Connor E, Greendale GA. Hyperkyphotic posture predicts mortality in older community-dwelling men and women: A prospective study. Journal of the American Geriatrics Society. 2004; 52(10):1662-7. [DOI:10.1111/j.1532-5415.2004.52458.x]

[3] Naderi E. [Does obesity affect the efficacy of therapeutic exercise on pain intensity and disability in patients with chronic non-specific low back pain (Persian)]. Journal of Applied Physics. 2017; 7(4):71-83.

[4] Ensrud KE, Black DM, Harris F, Ettinger B, Cummings SR. Correlates of kyphosis in older women. Journal of the American Geriatrics Society. 1997; 45(6):682-7. [DOI:10.1111/j.1532-5415.1997. tb01470.x] [PMID]

[5] Yalfani A, Anbarian M, Nikoo R, Naderi A. [Relationship between postural control with Sway-back malalignment in the nonathlete males (Persian)]. Journal of Ilam University of Medical Science. 2014; 22 (5):189-201.

[6] Renno ACM, Granito RN, Costa D, Oishi J, Driusso P. Effects of an exercise program on respiratory function, posture and on quality of life in osteoporotic women: A pilot study. Physiotherapy. 2005; 91(2):113-8. [DOI:10.1016/j.physio.2004.09.020]

[7] Hongo M, Itoi E, Sinaki M, Miyakoshi N, Shimada Y, Maekawa $\mathrm{S}$, et al. Effect of low-intensity back exercise on quality of life and back extensor strength in patients with osteoporosis: A randomized controlled trial. Osteoporosis International. 2007; 18(10):1389-95. [DOI:10.1007/s00198-007-0398-9]

[8] Zeng Y, Chen Z, Ma D, Guo Z, Qi Q, Li W, et al. The influence of kyphosis correction surgery on pulmonary function and thoracic volume. The Spine Journal. 2014; 39(21):1777-84. [DOI:10.1097/ BRS.0000000000000524]

[9] Culham GE, Hilda AIJ, Cheryl EK. Thoracic kyphosis, rib mobility, and lung volumes in normal women and women with osteoporosis. The Spine Journal. 1994; 19(11):1250-5. [DOI:10.1097/00007632-199405310-00010]

[10] Lorbergs AL, O'Connor GT, Zhou Y, Travison TG, Kiel DP, Cupples LA, et al. Severity of kyphosis and decline in lung function: The framingham study. The Journals of Gerontology. 2017; 72(5):689-94. [DOI:10.1093/gerona/glw124]

[11] Naderi A, Shaabani F, Malki F, Khosravi F. [Kinematic changes of body alignment resulting from backpack weight, location and carrying duration in 10 to 12 years old boy schoolchildren (Persian)]. Journal of Applied Sport Physiology. 2017; 13(25):25-36. [DOI: 10.22080/jaep.2017.1585]

[12] Sinaki M, Lynn SG. Reducing the risk of falls through proprioceptive dynamic posture training in osteoporotic women with kyphotic posturing: A randomized pilot study. American Journal of Physical Medicine \& Rehabilitation. 2002; 81(4):241-6. [DOI:10.1097/00002060-200204000-00001] [PMID]

[13] Fechtenbaum J, Etcheto A, Kolta S, Feydy A, Roux C, Briot K. Sagittal balance of the spine in patients with osteoporotic vertebral fractures. Osteoporosis International. 2016; 27(2):559-67. [DOI:10.1007/s00198-015-3283-y]
[14] Ishikawa Y, Miyakoshi N, Kasukawa Y, Hongo M, Shimada Y. Spinal curvature and postural balance in patients with osteoporosis. Osteoporosis International. 2009; 20(12):2049-53. [DOI:10.1007/s00198-009-0919-9]

[15] Eum R, Leveille SG, Kiely DK, Kiel DP, Samelson EJ, Bean JF. Is kyphosis related to mobility, balance and disability. American Journal of Physical Medicine \& Rehabilitation. 2013; 92(11):980-9. [DOI:10.1097/PHM.0b013e31829233ee]

[16] Sinaki M, McPhee MC, Hodgson SF, Offord KP. Relationship between bone mineral density of spine and strength of back extensors in healthy postmenopausal women. IMayo Clinic Proceedings. 1986; 61(2):116-22. [DOI:10.1016/S00256196(12)65197-0]

[17] Hongo M, Itoi E, Sinaki M, Miyakoshi N, Shimada Y, Maekawa $\mathrm{S}$, et al. Effect of low-intensity back exercise on quality of life and back extensor strength in patients with osteoporosis: A randomized controlled trial. Osteoporosis International. 2007; 18(10):1389-95. [DOI:10.1007/s00198-007-0398-9]

[18] Lombardi JRI, Oliveira LM, Monteiro CR, Confessor YQ, Barros TL, Natour J. Evaluation of physical capacity and quality of life in osteoporotic women. Osteoporosis International. 2004; 15(1):80-5. [DOI:10.1007/s00198-003-1512-2]

[19] Sinaki M, Itoi E, Rogers JW, Bergstralh EJ, Wahner HW. Correlation of back extensor strength with thoracic kyphosis and lumbar lordosis in estrogen-deficient women. American Journal of Physical Medicine \& Rehabilitation. 1995; 75(5):370-4. [DOI:10.1097/00002060-199609000-00013]

[20] Balouchy R, Ghiasi A, Naderi E, Sodoghi H. [The survey of cawthorne and cooksey exercise on the quality of life, balance and fatigue in patients with multiple sclerosis (Persian)]. Journal of Ilam University of Medical Science. 2014; 21(7):43-53.

[21] Greendale GA, McDivit A, Carpenter A, Seeger L, Huang MH. Yoga for women with hyperkyphosis: Results of a pilot study. American Journal of Public Health. 2002; 92(10):1611-4. [DOI:10.2105/AJPH.92.10.1611] [PMID] [PMCID]

[22] Pfeifer M, Begerow B, Minne HW. Effects of a new spinal orthosis on posture, trunk strength, and quality of life in women with postmenopausal osteoporosis: A randomized trial. American Journal of Physical Medicine \& Rehabilitation. 2004; 83(3):177-86. [DOI:10.1097/01.PHM.0000113403.16617.93] [PMID]

[23] Itoi E, Mehrsheed S, Sinaki M. Effect of back-strengthening exercise on posture in healthy women 49 to 65 years of age. Mayo Clinic Proceedings. 1994; 69(11):1054-9. [DOI:10.1016/S00256196(12)61372-X]

[24] Katzman WB, Sellmeyer DE, Stewart AL, Wanek L, Hamel KA. Changes in flexed posture, musculoskeletal impairments, and physical performance after group exercise in community-dwelling older women. Archives of Physical Medicine and Rehabilitation. 2007; 88(2):192-9. [DOI:10.1016/j.apmr.2006.10.033]

[25] Hoppenfeld S, Hutton R, Thomas H. Physical examination of the spine and extremities. New York: Appelton-CenturyCrofts; 1976

[26] Khalkhali M, Parnianpour M, Karimi H, Mobini B, Kazemnejhad A. The validity and reliability of measurement of thoracic kyphosis using flexible ruler in postural hyper-kyphotic patients. Journal of Rehabilitation. 2003; 4(3):18-23. [DOI.org/10.1016/ S0021-9290(06)85226-7] 
[27] Youdas JW, Garrett TR, Egan KS, Therneau TM. Lumbar lordosis and pelvic inclination in adults with chronic low back pain. Physical Therapy. 2000; 80(3):261-75. [PMID]

[28] Murray HC, Elliott C, Barton SE, Murray A. Do patients with ankylosing spondylitis have poorer balance than normal subjects. Rheumatology. 2000; 39(5):497-500. [DOI:10.1093/rheumatology/39.5.497] [PMID]

[29] De Oliveira TS, Candotti CT, La Torre M, Pelinson PP, Furlanetto TS, Kutchak FM, et al. Validity and reproducibility of the measurements obtained using the flexicurve instrument to evaluate the angles of thoracic and lumbar curvatures of the spine in the sagittal plane. Rehabilitation Research and Practice. 2012; 59(5):651-7. [DOI:10.1155/2012/186156]

[30] Brazier JE, Harper R, Jones NM, O'cathain A, Thomas KJ, Usherwood T, et al. Validating the SF-36 health survey questionnaire: New outcome measure for primary care. British Medical Journal. 1992; 305(6846):160-4. [DOI:10.1136/bmj.305.6846.160] [PMID] [PMCID]

[31] Montazeri A, Goshtasebi A, Vahdani Nia MS. The Short Form health survey (SF-36): Translation and validation study of the Iranian version. Journal of The Iranian Institute For Health Sciences Research. 2005; 5(1):49-56. [PMID]

[32] Franchignoni F, Tesio L, Martino MT, Ricupero C. Reliability of four simple, quantitative tests of balance and mobility in healthy elderly females. Aging Clinical and Experimental Research. 1998; 10(1):26-31. [DOI:10.1007/BF03339630]

[33] Steffen T, Seney M. Test-retest reliability and minimal detectable change on balance and ambulation tests, the 36-item shortform health survey, and the unified Parkinson disease rating scale in people with parkinsonism. Physical therapy. 2008; 88(6):733-46. [DOI:10.2522/ptj.20070214]

[34] Steffen TM, Hacker TA, Mollinger L. Age-and gender-related test performance in community-dwelling elderly people: SixMinute Walk Test, Berg Balance Scale, Timed Up \& Go Test, and gait speeds. Physical Therapy. 2002; 82(2):128-37. [DOI:10.1093/ ptj/82.2.128] [PMID]

[35] Hamilton DM, Haennel RG. Validity and reliability of the 6-minute walk test in a cardiac rehabilitation population. Journal of Cardiopulmonary Rehabilitation and Prevention. 2000; 20(3):156-64. [DOI:10.1097/00008483-200005000-00003]

[36] Lynch SS, Thigpen CA, Mihalik JP, Prentice WE, Padua D. Forward head and rounded shoulder postures in elite swimmers The effects of an exercise intervention on forward head and rounded shoulder postures in elite swimmers. British Journal of Sports Medicine. 2010; 44:376-81. [DOI:10.1136/bjsm.2009.066837]

[37] Seidi F, Rajabi R, Ebrahimi I, Alizadeh MH, Minoonejad H. The efficiency of corrective exercise interventions on thoracic hyperkyphosis angle. Journal of Back and Musculoskeletal Rehabilitation. 2013; 27(1):7-16. [DOI:10.3233/BMR-130411]

[38] Vaughn D, Brown E. The influence of an in-home based therapeutic exercise program on thoracic kyphosis angles. Journal of Back and Musculoskeletal Rehabilitation. 2007; 20(4):155-65. [DOI:10.3233/BMR-2007-20404]

[39] Imagama S, Matsuyama Y, Hasegawa Y, Sakai Y, Ito Z, Ishiguro $\mathrm{N}$, et al. Back muscle strength and spinal mobility are predictors of quality of life in middle-aged and elderly males. European Spine Journal. 2011; 20(6):954-61. [DOI:10.1007/s00586-010-1606-4]
[40] Hongo M, Miyakoshi N, Shimada Y, Sinaki M. Association of spinal curve deformity and back extensor strength in elderly women with osteoporosis in Japan and the United States. Osteoporosis International. 2012; 23(3):1029-34. [DOI:10.1007/ s00198-011-1624-z]

[41] Lonner B, Yoo A, Terran JS, Sponseller P, Samdani A, Betz $\mathrm{R}$, et al. Effect of spinal deformity on adolescent quality of life Comparison of operative scheuermann kyphosis, adolescen idiopathic scoliosis, and normal controls. The Spine Journal 2013; 38(12):1049-55. [DOI:10.1097/BRS.0b013e3182893c01]

[42] Naderi A, Baloochi R, Jabbari F, Eslami R. [Comparison between the effects of core stability exercises and neuromuscular exercises on dynamic balance and lower limb function of athletes with functional ankle instability (Persian)]. Scientific Journal of Kurdistan University of Medical Sciences. 2016; 21(4):61-72.

[43] Ryan SD, Fried LP. The impact of kyphosis on daily functioning. Journal of the American Geriatrics Society. 1997; 45(12):147986. [DOI:10.1111/j.1532-5415.1997.tb03199.x] [PMID]

[44] Lynn SG, Sinaki M, Westerlind KC. Balance characteristics of persons with osteoporosis. Archives of Physical Medicine and Rehabilitation. 1997; 78(3):273-7. [DOI:10.1016/S00039993(97)90033-2]

[45] Goodman C, Helgeson K. Exercise prescription for medical conditions: Handbook for physical therapists. Philadelphia Pennsylvania: FA Davis; 2010.

[46] Kasukawa Y, Miyakoshi N, Hongo M, Ishikawa Y, Noguch $\mathrm{H}$, Kamo K, et al. Relationships between falls, spinal curvature, spinal mobility and back extensor strength in elderly people. Journal of Bone and Mineral Metabolism. 2010; 28(1):82-7. [DOI:10.1007/s00774-009-0107-1]

[47] Ghanbarzadeh M, Mehdipour AR. Study and influence of exercise program on respiratory function of adults with kyphosis. Acta of Bioengineering and Biomechanics. 2009; 11(1):11-7. [PMID]

[48] Yalfani A, Naderi E, Shayesterudi Y. [Comparing the effectiveness of hydrotherapy and physiotherapy in the management of knee osteoarthritis (Persian)]. Journal of Research in Rehabilitation Science. 2012; 8(2):328-36.

[49] Shumway-cook A, Gruber W, Baldwin M, Liao S. The effect of multidimensional exercises on balance, mobility, and fall risk in community-dwelling older adults. Physical Therapy. 1997; 77(1):46-57. [DOI:10.1093/ptj/77.1.46] [PMID]

[50] O'Brien K, Culham E, Pickles B. Balance and skeletal alignment in a group of elderly female fallers and nonfallers. The Journals of Gerontology. 1997; 52(4):B221-6. [PMID: 9224428]

[51] Leech JA, Dulberg C, Kellie S, Pattee L, Gay J. Relationship of lung function to severity of osteoporosis in women 1-3. The American Review of Respiratory Disease. 1990; 141(1):68-71 [DOI:10.1164/ajrccm/141.1.68]

[52] Naderi A. [The comparison of effects 3 corrective exercise methods on the spinal alignment of the individuals with sway back posture. (Persian)]. Journal of Applied Exercise Physiology. 2018; 14(27):29-48. 
This Page Intentionally Left Blank 